\title{
Interval-Valued Hesitant Fuzzy Multiattribute Group Decision Making Based on Improved Hamacher Aggregation Operators and Continuous Entropy
}

\author{
Jun Liu, ${ }^{1}$ Ning Zhou, ${ }^{2}$ Li-Hua Zhuang, ${ }^{1}$ Ning Li, ${ }^{1}$ and Fei-Fei Jin ${ }^{1}$ \\ ${ }^{1}$ School of Information Science \& Engineering, Changzhou University, Changzhou, Jiangsu 213164, China \\ ${ }^{2}$ School of Petroleum Engineering, Changzhou University, Changzhou, Jiangsu 213164, China \\ Correspondence should be addressed to Ning Zhou; zhouning@cczu.edu.cn
}

Received 2 January 2017; Revised 21 April 2017; Accepted 26 April 2017; Published 26 September 2017

Academic Editor: Peide Liu

Copyright (C) 2017 Jun Liu et al. This is an open access article distributed under the Creative Commons Attribution License, which permits unrestricted use, distribution, and reproduction in any medium, provided the original work is properly cited.

Under the interval-valued hesitant fuzzy information environment, we investigate a multiattribute group decision making (MAGDM) method with continuous entropy weights and improved Hamacher information aggregation operators. Firstly, we introduce the axiomatic definition of entropy for interval-valued hesitant fuzzy elements (IVHFEs) and construct a continuous entropy formula on the basis of the continuous ordered weighted averaging (COWA) operator. Then, based on the Hamacher $t$-norm and $t$-conorm, the adjusted operational laws for IVHFEs are defined. In order to aggregate interval-valued hesitant fuzzy information, some new improved interval-valued hesitant fuzzy Hamacher aggregation operators are investigated, including the improved interval-valued hesitant fuzzy Hamacher ordered weighted averaging (I-IVHFHOWA) operator and the improved interval-valued hesitant fuzzy Hamacher ordered weighted geometric (I-IVHFHOWG) operator, the desirable properties of which are discussed. In addition, the relationship among these proposed operators is analyzed in detail. Applying the continuous entropy and the proposed operators, an approach to MAGDM is developed. Finally, a numerical example for emergency operating center (EOC) selection is provided, and comparative analyses with existing methods are performed to demonstrate that the proposed approach is both valid and practical to deal with group decision making problems.

\section{Introduction}

Fuzzy sets (FSs) [1] originally put forward by Zadeh are a very useful tool and have achieved a great success in various fields. Atanassov proposed the intuitionistic fuzzy sets (IFSs) [24], which are a generalization of the FSs. The introduction of IFSs proves to be very meaningful and practical and has been found to be highly useful to deal with vagueness [512]. Atanassov and Gargov further introduced the concept of interval-valued intuitionistic fuzzy sets (IVIFSs) [13] as a generalization of that of IFSs, whose components are intervals rather than exact numbers. Under some conditions, the decision makers (DMs) are usually irresolute and hesitant for one thing or another, which makes it difficult to determine the membership of an element to a set due to doubts among a few different values. In this case, Torra and Narukawa [14] and Torra [15] introduced the hesitant fuzzy set (HFS), which permits the membership having a collection of possible values. Due to the fact that the interval-valued fuzzy set (IVFS) [16] is usually more adequate or sufficient to real-life group decision making (GDM) problems than real numbers, Chen et al. $[17,18]$ proposed the interval-valued hesitant fuzzy set (IVHFS), which permits the membership having a collection of possible interval-valued numbers.

Since IVHFS was introduced, it has been used to deal with many problems, especially the MAGDM problems, one of the ways is aggregating the DMs' opinions under each attribute for alternatives and then obtaining the collective of attribute values for each alternative [19]. Based on the arithmetic aggregation methods [20-27], $\mathrm{Xu}$ [28] and $\mathrm{Xu}$ and Yager [29] investigated several new intuitionistic fuzzy arithmetic aggregation operators and intuitionistic fuzzy geometric aggregation operators. Wei et al. [30] proposed some intervalvalued hesitant fuzzy aggregation operators: interval-valued 
hesitant fuzzy weighted averaging (IVHFWA) operator, interval-valued hesitant fuzzy ordered weighted averaging (IVHFOWA) operator, interval-valued hesitant fuzzy weighted geometric (IVHFWG) operator, interval-valued hesitant fuzzy ordered weighted geometric (IVHFOWG) operator, interval-valued hesitant fuzzy power aggregation operators, interval-valued hesitant fuzzy prioritized weighted average (IVHFPWA) operator, and interval-valued hesitant fuzzy prioritized weighted geometric (IVHFPWG) operator. Then, some of their desirable properties were investigated in detail. Zhu et al. [31] developed the interval-valued hesitant fuzzy Einstein Choquet ordered averaging (IVHFECOA) operator and the interval-valued hesitant fuzzy Einstein Choquet ordered geometric (IVHFECOG) operator; then they applied these two operators to deal with multiattribute decision making problems. Hamacher $t$-conorm and $t$-norm [32] are proposed by Hamacher, which are more general and more flexible $[32,33]$. In this paper, we extend the Hamacher $t$-conorm and $t$-norm to interval-valued hesitant fuzzy environment and investigate some improved intervalvalued hesitant fuzzy Hamacher operators that allow DMs to have more choice in MAGDM problems.

Entropy is one of the important research topics in the fuzzy theory, which has been widely used in practical applications [34]. Zadeh first introduced the concept of fuzzy entropy [35]. Moreover, De Luca and Termini [36] presented the axioms with which the fuzzy entropy should comply and defined the entropy for a FS. Based on the ratio of intuitionistic fuzzy cardinalities, Szmidt and Kacprzyk [37] have given the axiomatic requirements of intuitionistic fuzzy entropy measure and proposed a nonprobabilistic-type entropy measure for IFSs. Ye [38] constructed two entropy measures for IVIFSs and established an entropy weighted model to determine the entropy weights with respect to a decision matrix provided as IVIFSs. Wei et al. [39] developed an entropy measure for IVIFSs, which generalized three entropy measures for IFSs. Xu and Xia [34] introduced the concepts of entropy and cross-entropy for HFS and discussed their desirable properties.

From the above analysis, we can see that IVHFS is a very useful tool to cope with uncertainty. More and more decision making methods and theories have been developed on the basis of IVHFSs. On the one hand, just as the HFSs, introducing the axiomatic definition of entropy and investigating some entropy formulas for IVHFSs are the important issues. On the other hand, more and more multiattribute GDM methods and theories have been developed with the Hamacher $t$-norm and $t$-conorm. However, there are few aggregation techniques to use the Hamacher operations on IVHFSs. Therefore, it is necessary and meaningful to study some issues. For example, what is it like the expression of the interval-valued hesitant fuzzy continuous entropy formula on the basis of COWA operator? What is the relationship among the new improved interval-valued hesitant fuzzy Hamacher aggregation operators?

In this paper, the axiomatic definition of entropy and an entropy formula for IVHFEs are investigated, and then some new improved Hamacher aggregation operators are proposed to aggregate interval-valued hesitant fuzzy information.
A MAGDM approach is developed, which is based on the entropy weights and the Hamacher information aggregation operators.

To do this, the rest of the paper is organized as follows. In Section 2, we briefly review some basic concepts, including IVHFSs, Hamacher $t$-norm, and $t$-conorm. Section 3 gives the axiomatic definition of entropy for IVHFEs and constructs an interval-valued hesitant fuzzy continuous entropy formula. In Section 4, the new adjusted operations for IVHFEs are presented, and we investigate some improved interval-valued hesitant fuzzy Hamacher information aggregation operators, which are followed by the discussion of the relationship among the proposed operators. Section 5 develops an approach to MAGDM with the continuous entropy formula and the proposed operators. In Section 6, we provide a numerical example of EOCs evaluation to illustrate the application of the developed method. Finally, we end the paper by summarizing the main conclusions in Section 7.

\section{Preliminaries}

In this section, we furnish a brief review on some basic concepts, including IVHFSs and Hamacher $t$-norm and $t$ conorm.

Chen et al. [17, 18] first introduced the concept of IVHFS, which is defined as follows.

Definition 1. Let $X=\left\{x_{1}, x_{2}, \ldots, x_{n}\right\}$ be a fixed set, let $D[0,1]$ be the set of all closed subintervals of $[0,1]$. An IVHFS on $X$ is defined as

$$
A=\left\{\left\langle x_{i}, \alpha_{A}\left(x_{i}\right)\right\rangle \mid x_{i} \in X\right\},
$$

where $\alpha_{A}\left(x_{i}\right): X \rightarrow D[0,1]$ is a set of all possible intervalvalued membership degrees of the element $x_{i} \in X$ to the set A. $\alpha_{i}=\alpha_{A}\left(x_{i}\right)=\left\{\gamma_{i} \mid \gamma_{i} \in \alpha_{A}\left(x_{i}\right)\right\}=\left\{\left[\gamma_{i}^{L}, \gamma_{i}^{U}\right] \mid \gamma_{i} \in\right.$ $\left.\alpha_{A}\left(x_{i}\right)\right\}$ is called the IVHFE; here $\gamma_{i}^{L}=\inf \gamma_{i}$ and $\gamma_{i}^{U}=\sup \gamma_{i}$ represent the lower and upper limits of $\gamma_{i}$, respectively. The complement of the IVHFE $\alpha$ denotes $\alpha^{c}=\left\{\left[1-\gamma^{U}, 1-\gamma^{L}\right]\right.$ | $\gamma \in \alpha$. Let $H$ be the set of all IVHFEs.

Remark 2. Notice that the number of values in different IVHFEs may be different. Suppose that $l_{\alpha}$ stands for the number of values in $\alpha$; then the following assumptions are made.

(R1) All the elements in each IVHFE $\alpha=\left\{\left[\gamma^{(1) L}, \gamma^{(1) U}\right]\right.$, $\left.\left[\gamma^{(2) L}, \gamma^{(2) U}\right], \ldots,\left[\gamma^{\left(l_{\alpha}\right) L}, \gamma^{\left(l_{\alpha}\right) U}\right]\right\}$ are arranged in decreasing order, and let $\left[\gamma^{(t) L}, \gamma^{(t) U}\right]$ be the $t$ th largest interval number in $\alpha$.

(R2) If $l_{\alpha} \neq l_{\beta}$, then $l=\max \left\{l_{\alpha}, l_{\beta}\right\}$. To have a correct comparison, the two IVHFEs $\alpha$ and $\beta$ should have the same length. If there are fewer elements in $\alpha$ than in $\beta$, an extension of $\alpha$ should be considered optimistically by repeating its maximum element until it has the same length with $\beta$.

(R3) For convenience, we assume that all the IVHFEs have the same length $l$.

In order to compare among the different IVHFEs, we first give the properties of interval numbers. 
Definition 3 (see [40]). Let $a=\left[a^{L}, a^{U}\right]$ and $b=\left[b^{L}, b^{U}\right]$ be two interval numbers; then

(1) $a=b \Leftrightarrow a^{L}=b^{L}$ and $a^{U}=b^{U}$;

(2) $a+b=\left[a^{L}+a^{U}, b^{L}+b^{U}\right]$;

(3) $\rho a=\left[\rho a^{L}, \rho a^{U}\right], \rho \geq 0$.

Definition 4 (see [40]). Let $a=\left[a^{L}, a^{U}\right]$ and $b=\left[b^{L}, b^{U}\right]$ be two interval numbers, and let $L_{a}=a^{U}-a^{L}>0$ and $L_{b}=$ $b^{U}-b^{L}>0$; then the degree of possibility of $a \geq b$ is defined as

$$
p(a \geq b)=\max \left\{1-\max \left(\frac{b^{U}-a^{L}}{L_{a}+L_{b}}, 0\right), 0\right\} .
$$

Definition 5 (see [17]). For an IVHFE $\alpha, s(\alpha)=(1 / l) \sum_{t}^{l} \gamma^{(t)}$ is called the score function of $\alpha$. For two IVHFEs $\alpha_{1}$ and $\alpha_{2}$, if $s\left(\alpha_{1}\right) \geq s\left(\alpha_{2}\right)$, then $\alpha_{1} \geq \alpha_{2}$.

In the following, we recall the triangular norm and conorm, which is an important notion in fuzzy set theory.

Definition 6 (see [41]). A function $T:[0,1]^{2} \rightarrow[0,1]$ is a $t$-norm if and only if it is commutative, associative, and nondecreasing and $T(x, 1)=x, \forall x \in[0,1]$.

The corresponding $t$-conorm of $S$ is the function $S$ : $[0,1]^{2} \rightarrow[0,1]$ defined by $S(x, y)=1-T(1-x, 1-y)$.

For many $t$-norms and $t$-conorms, there are some basic $t$-norms and $t$-conorms, including Algebraic product $T_{A}$, Algebraic sum $S_{A}$, Einstein product $T_{E}$, and Einstein sum $S_{E}$. Hamacher given the following generalized $t$-norm and $t$ conorm denoted the Hamacher $t$-norm and $t$-conorm [42]:

$$
\begin{array}{ll}
T(x, y)=\frac{x y}{\xi+(1-\xi)(x+y-x y)}, & \xi>0, \\
S(x, y)=\frac{x+y-x y-(1-\xi) x y}{1-(1-\xi) x y}, & \xi>0 .
\end{array}
$$

In particular, when $\xi=1$, then the Hamacher $t$-norm and $t$-conorm are reduced to the Algebraic product $T_{A}$ and Algebraic sum $S_{A}$; when $\xi=2$, then Hamacher $t$-norm and $t$ conorm are reduced to the Einstein product $T_{E}$ and Einstein sum $S_{E}$.

By using Hamacher $t$-conorm and $t$-norm, Li and Peng [43] introduced some operational laws for IVHFEs as follows.

Definition 7 (see [43]). Let $\alpha, \alpha_{1}$, and $\alpha_{2}$ be three IVHFEs; then

$$
\begin{aligned}
& \text { (1) } \alpha_{1} \oplus \alpha_{2}=\left\{\left[\frac{\gamma_{1}^{L}+\gamma_{2}^{L}-\gamma_{1}^{L} \gamma_{2}^{L}-(1-\xi) \gamma_{1}^{L} \gamma_{2}^{L}}{1-(1-\xi) \gamma_{1}^{L} \gamma_{2}^{L}},\right.\right. \\
& \left.\frac{\gamma_{1}^{U}+\gamma_{2}^{U}-\gamma_{1}^{U} \gamma_{2}^{U}-(1-\xi) \gamma_{1}^{U} \gamma_{2}^{U}}{1-(1-\xi) \gamma_{1}^{L} \gamma_{2}^{L}}\right] \mid \gamma_{1} \in \alpha_{1}, \gamma_{2}
\end{aligned}
$$

$$
\left.\in \alpha_{2}\right\}
$$

(2) $\alpha_{1} \otimes \alpha_{2}=\left\{\left[\frac{\gamma_{1}^{L} \gamma_{2}^{L}}{\xi+(1-\xi)\left(\gamma_{1}^{L}+\gamma_{2}^{L}-\gamma_{1}^{L} \gamma_{2}^{L}\right)}\right.\right.$,

$$
\left.\left.\frac{\gamma_{1}^{U} \gamma_{2}^{U}}{\xi+(1-\xi)\left(\gamma_{1}^{U}+\gamma_{2}^{U}-\gamma_{1}^{U} \gamma_{2}^{U}\right)}\right] \mid \gamma_{1} \in \alpha_{1}, \gamma_{2} \in \alpha_{2}\right\} \text {; }
$$

(3) $\rho \alpha=\left\{\left[\frac{\left(1+(\xi-1) \gamma^{L}\right)^{\rho}-\left(1-\gamma^{L}\right)^{\rho}}{\left(1+(\xi-1) \gamma^{L}\right)^{\rho}+(\xi-1)\left(1-\gamma^{L}\right)^{\rho}}\right.\right.$,

$$
\left.\left.\frac{\left(1+(\xi-1) \gamma^{U}\right)^{\rho}-\left(1-\gamma^{U}\right)^{\rho}}{\left(1+(\xi-1) \gamma^{U}\right)^{\rho}+(\xi-1)\left(1-\gamma^{U}\right)^{\rho}}\right] \mid \gamma \in \alpha\right\},
$$

$$
\rho>0 \text {; }
$$

(4) $\alpha^{\rho}=\left\{\left[\frac{\xi\left(\gamma^{L}\right)^{\rho}}{\left(1+(\xi-1)\left(1-\gamma^{L}\right)\right)^{\rho}+(\xi-1)\left(\gamma^{L}\right)^{\rho}}\right.\right.$,

$$
\begin{array}{r}
\left.\left.\frac{\xi\left(\gamma^{U}\right)^{\rho}}{\left(1+(\xi-1)\left(1-\gamma^{U}\right)\right)^{\rho}+(\xi-1)\left(\gamma^{U}\right)^{\rho}}\right] \mid \gamma \in \alpha\right\}, \\
\rho>0 .
\end{array}
$$

Based on the above operations, Li and Peng [43] developed a series of specific aggregation operators for IVHFEs.

Definition 8 (see [43]). Let $\alpha_{j}=\left\{\left[\gamma_{j}^{L}, \gamma_{j}^{U}\right] \mid \gamma_{j} \in \alpha_{j}\right\}(j=1,2$, $\ldots, n)$ be a collection of IVHFEs, and $W=\left(w_{1}, w_{2}, \ldots, w_{n}\right)^{T}$ is the associated weight vector of $\alpha_{j}(j=1,2, \ldots, n)$, with $0 \leq w_{j} \leq 1, \quad j=1,2, \ldots, n$ and $\sum_{j=1}^{n} w_{j}=1$. Then an intervalvalued hesitant fuzzy Hamacher ordered weighted averaging (IVHFHOWA) operator is a mapping IVHFHOWA $: H^{n} \rightarrow$ $H$, such that

$$
\begin{aligned}
& \operatorname{IVHFHOWA}\left(\alpha_{1}, \alpha_{2}, \ldots, \alpha_{n}\right)=\bigoplus_{j=1}^{n} w_{j} \alpha_{(j)} \\
& =\left\{\left[\frac{\prod_{j=1}^{n}\left(1+(\xi-1) \gamma_{(j)}^{L}\right)^{w_{j}}-\prod_{j=1}^{n}\left(1-\gamma_{(j)}^{L}\right)^{w_{j}}}{\prod_{j=1}^{n}\left(1+(\xi-1) \gamma_{(j)}^{L}\right)^{w_{j}}+(\xi-1) \prod_{j=1}^{n}\left(1-\gamma_{(j)}^{L}\right)^{w_{j}}},\right.\right. \\
& \left.\frac{\prod_{j=1}^{n}\left(1+(\xi-1) \gamma_{(j)}^{U}\right)^{w_{j}}-\prod_{j=1}^{n}\left(1-\gamma_{(j)}^{U}\right)^{w_{j}}}{\prod_{j=1}^{n}\left(1+(\xi-1) \gamma_{(j)}^{U}\right)^{w_{j}}+(\xi-1) \prod_{j=1}^{n}\left(1-\gamma_{(j)}^{U}\right)^{w_{j}}}\right] \mid \\
& \left.\quad \gamma_{1} \in \alpha_{1}, \gamma_{2} \in \alpha_{2}, \ldots, \gamma_{n} \in \alpha_{n}\right\},
\end{aligned}
$$

where $(j):\{1,2, \ldots, n\} \rightarrow\{1,2, \ldots, n\}$ is a permutation such that $\alpha_{(1)} \geq \alpha_{(2)} \geq \cdots \geq \alpha_{(n)}$.

Definition 9 (see [43]). Let $\alpha_{j}=\left\{\left[\gamma_{j}^{L}, \gamma_{j}^{U}\right] \mid \gamma_{j} \in \alpha_{j}\right\}(j=1,2$, $\ldots, n)$ be a collection of IVHFEs, and $W=\left(w_{1}, w_{2}, \ldots, w_{n}\right)^{T}$ is the associated weight vector of $\alpha_{j}(j=1,2, \ldots, n)$, with 
$0 \leq w_{j} \leq 1, j=1,2, \ldots, n$ and $\sum_{j=1}^{n} w_{j}=1$. Then an intervalvalued hesitant fuzzy Hamacher ordered weighted geometric (IVHFHOWG) operator is a mapping IVHFHOWG : $H^{n} \rightarrow$ $H$, such that

$$
\begin{aligned}
& \text { IVHFHOWG }\left(\alpha_{1}, \alpha_{2}, \ldots, \alpha_{n}\right)=\bigotimes_{j=1}^{n} \alpha_{(j)}^{w_{j}} \\
& =\left\{\left[\frac{\xi \prod_{j=1}^{n}\left(\gamma_{(j)}^{L}\right){ }^{w_{j}}}{\prod_{j=1}^{n}\left(1+(\xi-1)\left(1-\gamma_{(j)}^{L}\right)\right)^{w_{j}}+(\xi-1) \prod_{j=1}^{n}\left(\gamma_{(j)}^{L}\right)^{w_{j}}},\right.\right. \\
& \left.\frac{\xi \prod_{j=1}^{n}\left(\gamma_{(j)}^{U}\right)^{w_{j}}}{\prod_{j=1}^{n}\left(1+(\xi-1)\left(1-\gamma_{(j)}^{U}\right)\right)^{w_{j}}+(\xi-1) \prod_{j=1}^{n}\left(\gamma_{(j)}^{U}\right)^{w_{j}}}\right] \mid \gamma_{1} \\
& \left.\quad \in \alpha_{1}, \gamma_{2} \in \alpha_{2}, \ldots, \gamma_{n} \in \alpha_{n}\right\},
\end{aligned}
$$

where $(j):\{1,2, \ldots, n\} \rightarrow\{1,2, \ldots, n\}$ is a permutation such that $\alpha_{(1)} \geq \alpha_{(2)} \geq \cdots \geq \alpha_{(n)}$.

Theorem 10 (idempotency, see [43], Theorems 19 and 24). Let $\alpha_{j}=\left\{\left[\gamma_{j}^{L}, \gamma_{j}^{U}\right] \mid \gamma_{j} \in \alpha_{j}\right\} \quad(j=1,2, \ldots, n)$ be a collection of IVHFEs, if all $\alpha_{j}, j=1,2, \ldots, n$, are equal: that is, $\alpha_{j}=\alpha$ for all $j$; then

$$
\begin{aligned}
& \operatorname{IVHFHOWA}\left(\alpha_{1}, \alpha_{2}, \ldots, \alpha_{n}\right)=\alpha, \\
& \operatorname{IVHFHOWG}\left(\alpha_{1}, \alpha_{2}, \ldots, \alpha_{n}\right)=\alpha .
\end{aligned}
$$

\section{Interval-Valued Hesitant Fuzzy Continuous Entropy}

In this section, we introduce the axiomatic definition of entropy for IVHFEs and then construct an interval-valued hesitant fuzzy continuous entropy formula on the basis of COWA operator.

The COWA operator was developed by Yager [44], which extends the OWA operator [20].

Definition 11. A COWA operator is a mapping $F: M \rightarrow$ $R^{+}$associated with a basic unit interval monotonic (BUM) function, $Q$, such that

$$
\begin{aligned}
F_{\mathrm{Q}}(a) & =F_{\mathrm{Q}}\left(\left[a^{L}, a^{U}\right]\right) \\
& =\int_{0}^{1} \frac{d Q(y)}{d y}\left(a^{U}-y\left(a^{U}-a^{L}\right)\right) d y,
\end{aligned}
$$

where $a=\left[a^{L}, a^{U}\right] \in M$ and $M$ is the set of all nonnegative interval numbers.

Denoting $\lambda=\int_{0}^{1} \mathrm{Q}(y) d y$, then we have

$$
F_{\mathrm{Q}}(a)=F_{\mathrm{Q}}\left(\left[a^{L}, a^{U}\right]\right)=\lambda a^{U}+(1-\lambda) a^{L},
$$

where $\lambda$ is the attitudinal character of $Q$. Thus, $F_{Q}\left(\left[a^{L}, a^{U}\right]\right)$ is the weighted average of the end points of the closed interval with attitudinal character parameter, and it is called the attitudinal expected value of $\left[a^{L}, a^{U}\right]$.

In what follows, we first present the axiomatic definition of entropy for IVHFEs and then investigate a continuous entropy formula for IVHFEs.

Definition 12. An entropy on IVHFE $\alpha=\left\{\left[\gamma^{(t) L}, \gamma^{(t) U}\right] \mid t=\right.$ $1,2, \ldots, l\}$ is an interval-valued function $E: H \rightarrow[0,1]$, which satisfies the following axiomatic requirements:

(E1) $E(\alpha)=0$, if and only if $\alpha=[0,0]$ or $\alpha=[1,1]$.

(E2) $E(\alpha)=1$, if and only if $\gamma^{(t) L}+\gamma^{(l-t+1) U}=1$, for $t=$ $1,2, \ldots, l$.

(E3) $E(\alpha)=E\left(\alpha^{c}\right)$.

(E4) $E(\alpha) \leq E(\beta)$, if

$\gamma^{(t) L} \leq \beta^{(t) L}$ and $\gamma^{(t) U} \leq \beta^{(t) U}$ when $\beta^{(t) L}+\beta^{(l-t+1) U} \leq$ 1 , for $t=1,2, \ldots, l$,

or

$$
\begin{aligned}
& \gamma^{(t) L} \geq \beta^{(t) L} \text { and } \gamma^{(t) U} \geq \beta^{(t) U} \text { when } \beta^{(t) L}+\beta^{(l-t+1) U} \geq \\
& 1, \text { for } t=1,2, \ldots, l .
\end{aligned}
$$

Note that if the IVHFE $\alpha=\left\{\left[\gamma^{(t) L}, \gamma^{(t) U}\right] \mid t=1,2, \ldots, l\right\}$ is reduced to a hesitant fuzzy element $h=\left\{\gamma^{(t)} \mid t=\right.$ $1,2, \ldots, l\}$, that is, all the interval numbers $\left[\gamma^{(t) L}, \gamma^{(t) U}\right]$ in $\alpha$ are reduced to crisp values $\gamma^{(t)}$, then the requirements of axiomatic definition of entropy for IVHFE are reduced to the following conditions:

(1) $E(h)=0$, if and only if $h=0$ or $h=1$.

(2) $E(h)=1$, if and only if $\gamma^{(t)}+\gamma^{(l-t+1)}=1$, for $t=1,2$, $\ldots, l$.

(3) $E(h)=E\left(h^{c}\right)$.

(4) $E(h) \leq E(g)$, if $\gamma^{(t)} \leq \beta^{(t)}$ for $\beta^{(t)}+\beta^{(l-t+1)} \leq 1$, or $\gamma^{(t)} \geq \beta^{(t)}$ for $\beta^{(t)}+\beta^{(l-t+1)} \geq 1, t=1,2, \ldots, l$.

The above conditions are equivalent to the requirements of the axiomatic definition of entropy for hesitant fuzzy elements proposed by Xu and Xia [34].

Based on the COWA operator, we construct an information measure formula for IVHFE $\alpha$ as follows:

$$
\begin{aligned}
E(\alpha) & =\frac{1}{(\sqrt{2}-1) l} \\
& \cdot \sum_{t=1}^{l}\left(\sqrt{2} \sin \frac{F_{\mathrm{Q}}\left(\gamma^{(t)}\right)+2-F_{\mathrm{Q}}\left(\gamma^{(t) c}\right)}{4} \pi-1\right) .
\end{aligned}
$$

In what follows, we prove that $E(\alpha)$ is an entropy measure of IVHFE $\alpha$.

Theorem 13. The mapping $E(\alpha)$, defined by (10), is an entropy measure for IVHFE $\alpha$.

Proof. In order for (10) to be qualified as a sensible measure of interval-valued hesitant fuzzy entropy, it must satisfy conditions (E1)-(E4) in Definition 9. 
Let $f(x)=(1 /(\sqrt{2}-1))(\sqrt{2} \sin (\pi / 4) x-1), x \in[1,3] ;$ then we have $d f(x) / d x=(\sqrt{2} \pi / 4(\sqrt{2}-1)) \cos (\pi / 4) x$.

If $x \in[1,2]$, then $d f(x) / d x \geq 0$, which means that $f(x)$ is an increasing function of $x$, for $x \in[1,2]$. If $x \in[2,3]$, then $d f(x) / d x \leq 0$, which means that $f(x)$ is a decreasing function of $x$, for $x \in[2,3]$. Since $f(x) \in[0,1]$, then $f_{\min }(x)=0$, if and only if $x=1$ or $x=3 ; f_{\max }(x)=1$, if and only if $x=2$.

(E1) If $\alpha=[0,0]$ or $\alpha=[1,1]$, then we obtain that

$$
\begin{aligned}
F_{\mathrm{Q}}\left(\gamma^{(t)}\right)+2-F_{\mathrm{Q}}\left(\gamma^{(t) c}\right) & =1 \\
\text { or } F_{\mathrm{Q}}\left(\gamma^{(t)}\right)+2-F_{\mathrm{Q}}\left(\gamma^{(t) c}\right) & =3,
\end{aligned}
$$

$$
t=1,2, \ldots, l .
$$

From the above analysis, we have $E(\alpha)=0$.

On the other hand, assume that $E(\alpha)=0$. Since $\alpha^{c}=$ $\left\{\gamma^{(1) c}, \gamma^{(2) c}, \ldots, \gamma^{(l) c}\right\}=\left\{\left[1-\gamma^{(l) U}, 1-\gamma^{(l) L}\right],\left[1-\gamma^{(l-1) U}, 1-\right.\right.$ $\left.\left.\gamma^{(l-1) L}\right], \ldots,\left[1-\gamma^{(1) U}, 1-\gamma^{(1) L}\right]\right\}$, then $\gamma^{(t) c}=\left[1-\gamma^{(l-t+1) U}, 1-\right.$ $\left.\gamma^{(l-t+1) L}\right]$. For all $t=1,2, \ldots, l$, we have

$$
\begin{aligned}
0 & \leq \gamma^{(t) L}=\lambda \gamma^{(t) L}+(1-\lambda) \gamma^{(t) L} \\
& \leq \lambda \gamma^{(t) U}+(1-\lambda) \gamma^{(t) L} \leq \lambda \gamma^{(t) U}+(1-\lambda) \gamma^{(t) U} \\
& =\gamma^{(t) U} \leq 1 .
\end{aligned}
$$

It follows that $F_{\mathrm{Q}}\left(\gamma^{(t)}\right) \in[0,1], t=1,2, \ldots, l$; then $F_{\mathrm{Q}}\left(\gamma^{(t)}\right)+$ $2-F_{Q}\left(\gamma^{(t) c}\right) \in[1,3], t=1,2, \ldots, l$. Therefore, every term in the summation of $E(\alpha)$ is nonnegative. Since $E(\alpha)=0$, then we deduce that every term should be zero in $E(\alpha)$; that is, for $\forall t \in\{1,2, \ldots, l\}$, we have

$$
\begin{aligned}
& \frac{1}{(\sqrt{2}-1)}\left(\sqrt{2} \sin \frac{F_{\mathrm{Q}}\left(\gamma^{(t)}\right)+2-F_{\mathrm{Q}}\left(\gamma^{(t) c}\right)}{4} \pi-1\right) \\
& \quad=0 .
\end{aligned}
$$

From the above analysis, we know that (13) holds for all $j$, if and only if $F_{\mathrm{Q}}\left(\gamma^{(t)}\right)+2-F_{\mathrm{Q}}\left(\gamma^{(t) c}\right)=1$ or $F_{\mathrm{Q}}\left(\gamma^{(t)}\right)+2-$ $F_{Q}\left(\gamma^{(t) c}\right)=3$, for $\forall \lambda \in[0,1], t=1,2, \ldots, l$.

(i) If $F_{Q}\left(\gamma^{(t)}\right)+2-F_{Q}\left(\gamma^{(t) c}\right)=1$, for $\forall \lambda \in[0,1], t=$ $1,2, \ldots, l$.

Let $\Phi_{t}(\lambda)=F_{\mathrm{Q}}\left(\gamma^{(t)}\right)+2-F_{\mathrm{Q}}\left(\gamma^{(t) c}\right)-1, t=1,2, \ldots, l$; then we obtain that

$$
\begin{aligned}
\Phi_{t}(\lambda) & =F_{\mathrm{Q}}\left(\gamma^{(t)}\right)+2-F_{\mathrm{Q}}\left(\gamma^{(t) c}\right)-1 \\
= & \left(\lambda \gamma^{(t) U}+(1-\lambda) \gamma^{(t) L}\right) \\
& -\left(\lambda\left(1-\gamma^{(l-t+1) L}\right)+(1-\lambda)\left(1-\gamma^{(l-t+1) U}\right)\right) \\
& +1 \\
= & \left(\gamma^{(t) U}-\gamma^{(t) L}+\gamma^{(l-t+1) L}-\gamma^{(l-t+1) U}\right) \lambda+\gamma^{(t) L} \\
& +\gamma^{(l-t+1) U} .
\end{aligned}
$$

Therefore, for $\forall \lambda \in[0,1], t=1,2, \ldots, l$, we have

$$
\begin{aligned}
& \left(\gamma^{(t) U}-\gamma^{(t) L}+\gamma^{(l-t+1) L}-\gamma^{(l-t+1) U}\right) \lambda+\gamma^{(t) L} \\
& +\gamma^{(l-t+1) U}=0 .
\end{aligned}
$$

then $\gamma^{(t) U}-\gamma^{(t) L}+\gamma^{(l-t+1) L}-\gamma^{(l-t+1) U}=0$ and $\gamma^{(t) L}+\gamma^{(l-t+1) U}=$ $0, t=1,2, \ldots, l$. As $\gamma^{(t) U}, \gamma^{(t) L}, \gamma^{(l-t+1) L}, \gamma^{(l-t+1) U} \in[0,1]$, $t=1,2, \ldots, l$, thus $\gamma^{(t) L}=\gamma^{(t) U}=0, t=1,2, \ldots, l$. Hence, $\alpha=[0,0]$.

(ii) If $F_{\mathrm{Q}}\left(\gamma^{(t)}\right)+2-F_{\mathrm{Q}}\left(\gamma^{(t) c}\right)=3$, for $\forall \lambda \in[0,1], t=$ $1,2, \ldots, l$. Since $F_{\mathrm{Q}}\left(\gamma^{(t)}\right) \in[0,1]$ and $F_{\mathrm{Q}}\left(\gamma^{(t)}\right)+2-F_{\mathrm{Q}}\left(\gamma^{(t) c}\right) \in$ $[1,3]$, for $\forall \lambda \in[0,1], t=1,2, \ldots, l$, and then we obtain that $F_{Q}\left(\gamma^{(t)}\right)=1, F_{Q}\left(\gamma^{(t) c}\right)=0$, for $\forall \lambda \in[0,1], t=1,2, \ldots, l$. According to (9), we have $\lambda \gamma^{(t) U}+(1-\lambda) \gamma^{(t) L}=1$, for $\forall \lambda \epsilon$ $[0,1], t=1,2, \ldots, l$; then $\gamma^{(t) L}=\gamma^{(t) U}=1, t=1,2, \ldots, l$. Hence, $\alpha=[1,1]$.

(E2) Suppose that $\gamma^{(t) L}+\gamma^{(l-t+1) U}=1$, for $t=1,2, \ldots, l$; then $\gamma^{(t) U}+\gamma^{(l-t+1) L}=1, t=1,2, \ldots, l$, and we have

$$
\begin{aligned}
& F_{\mathrm{Q}}\left(\gamma^{(t)}\right)+2-F_{\mathrm{Q}}\left(\gamma^{(t) c}\right) \\
&=\left(\lambda \gamma^{(t) U}+(1-\lambda) \gamma^{(t) L}\right)+2 \\
&-\left(\lambda\left(1-\gamma^{(l-t+1) L}\right)+(1-\lambda)\left(1-\gamma^{(l-t+1) U}\right)\right) \\
&= \lambda\left(\gamma^{(t) U}+\gamma^{(l-t+1) L}\right)+(1-\lambda)\left(\gamma^{(t) L}+\gamma^{(l-t+1) U}\right) \\
&+1=\lambda+(1-\lambda)+1=2 .
\end{aligned}
$$

From (10), we obtain that

$$
E_{1}(\alpha)=\frac{1}{(\sqrt{2}-1) l} \sum_{j=1}^{l}\left(\sqrt{2} \sin \frac{2}{4} \pi-1\right)=1 .
$$

On the other hand, from the above analysis, we have $F_{Q}\left(\gamma^{(t)}\right) \in[0,1]$ and $F_{Q}\left(\gamma^{(t)}\right)+2-F_{Q}\left(\gamma^{(t) c}\right) \in[1,3]$, and it is obvious that $0 \leq E(\alpha) \leq 1$. If $E(\alpha)=1$, then

$$
F_{\mathrm{Q}}\left(\gamma^{(t)}\right)+2-F_{\mathrm{Q}}\left(\gamma^{(t) c}\right)=2,
$$

$$
\text { for } \forall \lambda \in[0,1], t=1,2, \ldots, l \text {. }
$$

Therefore,

$$
\begin{aligned}
\left(\gamma^{(t) U}\right. & \left.-\gamma^{(t) L}+\gamma^{(l-t+1) L}-\gamma^{(l-t+1) U}\right) \lambda+\gamma^{(t) L} \\
& +\gamma^{(l-t+1) U}-1=0,
\end{aligned}
$$

for $\forall \lambda \in[0,1], t=1,2, \ldots, l$;

it follows that $\gamma^{(t) U}-\gamma^{(t) L}+\gamma^{(l-t+1) L}-\gamma^{(l-t+1) U}=0$ and $\gamma^{(t) L}+$ $\gamma^{(l-t+1) U}-1=0, t=1,2, \ldots, l$; that is,

$$
\gamma^{(t) L}+\gamma^{(l-t+1) U}=1, \quad t=1,2, \ldots, l .
$$

(E3) As $\alpha^{c}=\left\{\left[1-\gamma^{(l) U}, 1-\gamma^{(l) L}\right],\left[1-\gamma^{(l-1) U}, 1-\right.\right.$ $\left.\left.\gamma^{(l-1) L}\right], \ldots,\left[1-\gamma^{(1) U}, 1-\gamma^{(1) L}\right]\right\}$, then $\left(\alpha^{c}\right)^{c}=\alpha$, and we have

$$
\begin{aligned}
& F_{\mathrm{Q}}\left(\gamma^{(t) c}\right)+2-F_{\mathrm{Q}}\left(\left(\gamma^{c}\right)^{(t) c}\right) \\
& \quad=F_{\mathrm{Q}}\left(\gamma^{(t) c}\right)+2-F_{\mathrm{Q}}\left(\gamma^{(t)}\right), \quad t=1,2, \ldots, l .
\end{aligned}
$$


Since $\sin (x+\pi / 2)=\sin (-x+\pi / 2), x \in R$, and then

$$
\begin{aligned}
E & \left(\alpha^{c}\right)=\frac{1}{(\sqrt{2}-1) l} \\
& \cdot \sum_{t=1}^{l}\left(\sqrt{2} \sin \frac{F_{\mathrm{Q}}\left(\gamma^{(t) c}\right)+2-F_{\mathrm{Q}}\left(\left(\gamma^{c}\right)^{(t) c}\right)}{4} \pi-1\right) \\
& =\frac{1}{(\sqrt{2}-1) l} \\
& \cdot \sum_{t=1}^{l}\left(\sqrt{2} \sin \frac{F_{\mathrm{Q}}\left(\gamma^{(t) c}\right)+2-F_{\mathrm{Q}}\left(\gamma^{(t)}\right)}{4} \pi-1\right) \\
& =\frac{1}{(\sqrt{2}-1) l} \\
& \cdot \sum_{t=1}^{l}\left(\sqrt{2} \sin \left(\frac{F_{\mathrm{Q}}\left(\gamma^{(t) c}\right)-F_{\mathrm{Q}}\left(\gamma^{(t)}\right)}{4} \pi+\frac{\pi}{2}\right)-1\right) \\
& =\frac{1}{(\sqrt{2}-1) l} \\
& \cdot \sum_{t=1}^{l}\left(\sqrt{2} \sin \left(-\frac{F_{\mathrm{Q}}\left(\gamma^{(t) c}\right)-F_{\mathrm{Q}}\left(\gamma^{(t)}\right)}{4} \pi+\frac{\pi}{2}\right)\right. \\
& \left.-1)=\frac{1}{(\sqrt{2}-1) l} \pi-1\right) \\
& \cdot \sum_{t=1}^{l}\left(\sqrt{2} \sin \frac{F_{\mathrm{Q}}\left(\gamma^{(t)}\right)+2-F_{\mathrm{Q}}\left(\gamma^{(t) c}\right)}{4} \pi(\alpha) .\right.
\end{aligned}
$$

(E4) Assume that $\gamma^{(t) L} \leq \beta^{(t) L}$ and $\gamma^{(t) U} \leq \beta^{(t) U}$ when $\beta^{(t) L}+\beta^{(l-t+1) U} \leq 1$, for $t=1,2, \ldots, l$; then

$$
\begin{aligned}
& \gamma^{(t) L}+\gamma^{(l-t+1) U} \leq \beta^{(t) L}+\beta^{(l-t+1) U} \leq 1, \\
& \gamma^{(t) U}+\gamma^{(l-t+1) L} \leq \beta^{(t) U}+\beta^{(l-t+1) L} \leq 1,
\end{aligned}
$$

$$
t=1,2, \ldots, l \text {. }
$$

It follows that

$$
\begin{aligned}
1 \leq & F_{\mathrm{Q}}\left(\gamma^{(t)}\right)+2-F_{\mathrm{Q}}\left(\gamma^{(t) c}\right) \\
= & \lambda\left(\gamma^{(t) U}+\gamma^{(l-t+1) L}\right)+(1-\lambda)\left(\gamma^{(t) L}+\gamma^{(l-t+1) U}\right) \\
& +1 \\
\leq & \lambda\left(\beta^{(t) U}+\beta^{(l-t+1) L}\right)+(1-\lambda)\left(\beta^{(t) L}+\beta^{(l-t+1) U}\right) \\
& +1=F_{\mathrm{Q}}\left(\beta^{(t)}\right)+2-F_{\mathrm{Q}}\left(\beta^{(t) c}\right),
\end{aligned}
$$

$$
\begin{aligned}
F_{\mathrm{Q}}\left(\beta^{(t)}\right)+2-F_{\mathrm{Q}}\left(\beta^{(t) c}\right) \\
=\lambda\left(\beta^{(t) U}+\beta^{(l-t+1) L}\right)+(1-\lambda)\left(\beta^{(t) L}+\beta^{(l-t+1) U}\right) \\
\quad+1 \leq \lambda+(1-\lambda)+1=2 .
\end{aligned}
$$

Thus,

$$
\begin{aligned}
1 & \leq F_{Q}\left(\gamma^{(t)}\right)+2-F_{Q}\left(\gamma^{(t) c}\right) \\
& \leq F_{Q}\left(\beta^{(t)}\right)+2-F_{Q}\left(\beta^{(t) c}\right) \leq 2 .
\end{aligned}
$$

Notice that $f(x)=(1 /(\sqrt{2}-1))(\sqrt{2} \sin (\pi / 4) x-1)$ is an increasing function of $x$, for $x \in[1,2]$; thus, we obtain that $E(\alpha) \leq E(\beta)$.

Similarly, if $\gamma^{(t) L} \geq \beta^{(t) L}$ and $\gamma^{(t) U} \geq \beta^{(t) U}$ when $\beta^{(t) L}+$ $\beta^{(l-t+1) U} \geq 1$, for $t=1,2, \ldots, l$, one can also prove that $E(\alpha) \leq$ $E(\beta)$.

Definition 14. Suppose that $F$ is a COWA operator associated with BUM function $Q$ and $\alpha$ is an IVHFE; then $E(\alpha)$, defined by (10), is called the continuous entropy on IVHFE $\alpha$.

\section{Improved Interval-Valued Hesitant Fuzzy Hamacher Information Aggregation Operators and the Relationship among the Proposed Operators}

In this section, we first point out that the IVHFHOWA operator and IVHFHOWG operator proposed by Li and Peng [43] do not satisfy the property of idempotency by employing an illustrative example, and then some new operational laws for IVHFEs are defined. Two improved interval-valued hesitant fuzzy Hamacher information aggregation operators are further investigated, including the I-IHHOWA operator and the I-IHHOWG operator. In addition, we analyze the relationship among these proposed operators.

\subsection{Improved Interval-Valued Hesitant Fuzzy Hamacher Information Aggregation Operators}

Example 15. Suppose that $\alpha_{1}$ and $\alpha_{2}$ are two IVHFEs, and $\alpha_{1}=\alpha_{2}=\alpha=\{[0.1,0.2],[0.3,0.5]\}, W=(0.5,0.5)^{T}$. Without loss of generality, take $\xi=1$. According to Definition 8 , it follows that

$$
\begin{aligned}
& \operatorname{IVHFHOWA}(\alpha, \alpha)=\operatorname{IVHFHOWA}\left(\alpha_{1}, \alpha_{2}\right)=\{[1 \\
& \left.-\prod_{j=1}^{2}\left(1-\gamma_{(j)}^{L}\right)^{w_{j}}, 1-\prod_{j=1}^{2}\left(1-\gamma_{(j)}^{U}\right)^{w_{j}}\right] \mid \gamma_{1} \in \alpha_{1}, \gamma_{2} \\
& \left.\in \alpha_{2}\right\}=\left\{\left[1-(1-0.1)^{0.3} \times(1-0.1)^{0.7}, 1\right.\right. \\
& \left.-(1-0.2)^{0.3} \times(1-0.2)^{0.7}\right],\left[1-(1-0.1)^{0.3}\right.
\end{aligned}
$$




$$
\begin{aligned}
& \left.\times(1-0.3)^{0.7}, 1-(1-0.2)^{0.3} \times(1-0.5)^{0.7}\right],[1 \\
& -(1-0.3)^{0.3} \times(1-0.1)^{0.7}, 1-(1-0.5)^{0.3} \\
& \left.\times(1-0.2)^{0.7}\right],\left[1-(1-0.3)^{0.3} \times(1-0.5)^{0.7}, 1\right. \\
& \left.\left.-(1-0.3)^{0.3} \times(1-0.5)^{0.7}\right]\right\}=\{[0.1000,0.2000], \\
& {[0.2452,0.4243],[0.1654,0.3052],} \\
& [0.3000,0.5000]\} .
\end{aligned}
$$

Let $\beta=\operatorname{IVHFHOWA}(\alpha, \alpha)$; then the score values of $\alpha$ and $\beta$ can be calculated as $s(\alpha)=[0.2,0.35], s(\beta)=$ $[0.2027,0.3574]$. Therefore, the degree of possibility of $s(\alpha) \geq$ $s(\beta)$ is

$$
\begin{aligned}
& p(s(\alpha) \geq s(\beta)) \\
& \quad=\max \left\{1-\max \left(\frac{0.3574-0.2}{0.15+0.1547}, 0\right), 0\right\}=0.4834 \\
& \quad<0.5 .
\end{aligned}
$$

Then we have $s(\beta)>s(\alpha)$; thus $\beta>\alpha$; that is,

$$
\operatorname{IVHFHOWA}(\alpha, \alpha)>\alpha \text {. }
$$

On the other hand, by the IVHFHOWG operator in Definition 9, it is obtained that

$$
\begin{aligned}
& \text { IVHFHOWG }(\alpha, \alpha)=\left\{\left[\prod_{j=1}^{2}\left(\gamma_{(j)}^{L}\right)^{w_{j}}, \prod_{j=1}^{2}\left(\gamma_{(j)}^{U}\right)^{w_{j}}\right] \mid \gamma_{1} \in \alpha_{1}, \gamma_{2}\right. \\
& \left.\in \alpha_{2}\right\}=\left\{\left[0.1^{0.3} \times 0.1^{0.7}, 0.2^{0.3} \times 0.2^{0.7}\right],\right. \\
& {\left[0.1^{0.3} \times 0.3^{0.7}, 0.2^{0.3} \times 0.5^{0.7}\right]} \\
& {\left[0.3^{0.3} \times 0.1^{0.7}, 0.5^{0.3} \times 0.2^{0.7}\right],} \\
& \left.\left[0.3^{0.3} \times 0.5^{0.7}, 0.3^{0.3} \times 0.5^{0.7}\right]\right\}=\{[0.1000,0.2000], \\
& {[0.2158,0.3798],[0.1390,0.2633],} \\
& [0.3000,0.5000]\} .
\end{aligned}
$$

Let $\chi=\operatorname{IVHFHOWG}(\alpha, \alpha)$; then the score value of $\chi$ is $s(\chi)=$ $[0.1887,0.3358]$. Therefore, the degree of possibility of $s(\alpha) \geq$ $s(\chi)$ is

$$
\begin{aligned}
& p(s(\alpha) \geq s(\chi)) \\
& \quad=\max \left\{1-\max \left(\frac{0.3358-0.2}{0.15+0.1471}, 0\right), 0\right\}=0.5429 \\
& \quad>0.5 .
\end{aligned}
$$

Then we have $s(\alpha)>s(\chi)$; thus, $\chi<\alpha$; that is,

$$
\operatorname{IVHFHOWG}(\alpha, \alpha)<\alpha \text {. }
$$

Example 15 demonstrates that Theorem 10 of the IVHFHOWA operator and IVHFHOWG operator cannot be tenable, which suffer from serious drawbacks. In this case, the operations on the IVHFEs need to be improved. In the following, some adjusted operations for IVHFEs are presented, and then two new improved intervalvalued hesitant fuzzy Hamacher aggregation operators are developed, which satisfy the properties of idempotency and boundedness.

Definition 16. Let $\alpha, \alpha_{1}$, and $\alpha_{2}$ be three IVHFEs; then

(1) $\alpha_{1} \dot{\oplus} \alpha_{2}$

$$
\begin{aligned}
& =\bigcup_{t=1}^{l}\left\{\left[\frac{\gamma_{1}^{(t) L}+\gamma_{2}^{(t) L}-\gamma_{1}^{(t) L} \gamma_{2}^{(t) L}-(1-\xi) \gamma_{1}^{(t) L} \gamma_{2}^{(t) L}}{1-(1-\xi) \gamma_{1}^{(t) L} \gamma_{2}^{(t) L}},\right.\right. \\
& \left.\left.\frac{\gamma_{1}^{(t) U}+\gamma_{2}^{(t) U}-\gamma_{1}^{(t) U} \gamma_{2}^{(t) U}-(1-\xi) \gamma_{1}^{(t) U} \gamma_{2}^{(t) U}}{1-(1-\xi) \gamma_{1}^{(t) U} \gamma_{2}^{(t) U}}\right]\right\} ;
\end{aligned}
$$

(2) $\alpha_{1} \dot{\otimes} \alpha_{2}$

$$
\begin{aligned}
& =\bigcup_{t=1}^{l}\left\{\left[\frac{\gamma_{1}^{(t) L} \gamma_{2}^{(t) L}}{\xi+(1-\xi)\left(\gamma_{1}^{(t) L}+\gamma_{2}^{(t) L}-\gamma_{1}^{(t) L} \gamma_{2}^{(t) L}\right)},\right.\right. \\
& \left.\left.\frac{\gamma_{1}^{(t) U} \gamma_{2}^{(t) U}}{\xi+(1-\xi)\left(\gamma_{1}^{(t) U}+\gamma_{2}^{(t) U}-\gamma_{1}^{(t) U} \gamma_{2}^{(t) U}\right)}\right]\right\} ; \\
& \text { (3) } \rho \alpha=\bigcup_{t=1}^{l}\left\{\left[\frac{\left(1+(\xi-1) \gamma^{(t) L}\right)^{\rho}-\left(1-\gamma^{(t) L}\right)^{\rho}}{\left(1+(\xi-1) \gamma^{(t) L}\right)^{\rho}+(\xi-1)\left(1-\gamma^{(t) L}\right)^{\rho}},\right.\right. \\
& \left.\left.\quad \frac{\left(1+(\xi-1) \gamma^{(t) U}\right)^{\rho}-\left(1-\gamma^{(t) U}\right)^{\rho}}{\left(1+(\xi-1) \gamma^{(t) U}\right)^{\rho}+(\xi-1)\left(1-\gamma^{(t) U}\right)^{\rho}}\right]\right\}, \quad \rho>0 ;
\end{aligned}
$$

(4) $\alpha^{\rho}$

$$
\begin{aligned}
& =\bigcup_{t=1}^{l}\left\{\left[\frac{\xi\left(\gamma^{(t) L}\right)^{\rho}}{\left(1+(\xi-1)\left(1-\gamma^{(t) L}\right)\right)^{\rho}+(\xi-1)\left(\gamma^{(t) L}\right)^{\rho}},\right.\right. \\
& \left.\left.\frac{\xi\left(\gamma^{(t) U}\right)^{\rho}}{\left(1+(\xi-1)\left(1-\gamma^{(t) U}\right)\right)^{\rho}+(\xi-1)\left(\gamma^{(t) U}\right)^{\rho}}\right]\right\},
\end{aligned}
$$

$$
\rho>0 .
$$

According to Definition 16, we obtain that $\alpha_{1}, \alpha_{2}, \alpha_{1} \dot{\oplus} \alpha_{2}$, and $\alpha_{1} \dot{\otimes} \alpha_{2}$ have the same length $l$. Based on the adjusted operational principle for IVHFEs, we develop the improved interval-valued hesitant fuzzy Hamacher aggregation operators as follows.

Definition 17. Let $\alpha_{j}=\left\{\left[\gamma_{j}^{L}, \gamma_{j}^{U}\right] \mid \gamma_{j} \in \alpha_{j}\right\}(j=1,2, \ldots, n)$ be a collection of IVHFEs; the improved IVHFHOWA (IIVHFHOWA) operator is a mapping I-IVHFHOWA : $H^{n} \rightarrow$ $H$, such that 


$$
\begin{aligned}
& \text { I-IVHFHOWA }\left(\alpha_{1}, \alpha_{2}, \ldots, \alpha_{n}\right)=\bigoplus_{j=1}^{n} w_{j} \alpha_{(j)}=\bigcup_{t=1}^{l}\left\{\left[\frac{\prod_{j=1}^{n}\left(1+(\xi-1) \gamma_{(j)}^{(t) L}\right)^{w_{j}}-\prod_{j=1}^{n}\left(1-\gamma_{(j)}^{(t) L}\right)^{w_{j}}}{\prod_{j=1}^{n}\left(1+(\xi-1) \gamma_{(j)}^{(t) L}\right)^{w_{j}}+(\xi-1) \prod_{j=1}^{n}\left(1-\gamma_{(j)}^{(t) L}\right)^{w_{j}}},\right.\right. \\
& \left.\left.\frac{\prod_{j=1}^{n}\left(1+(\xi-1) \gamma_{(j)}^{(t) U}\right)^{w_{j}}-\prod_{j=1}^{n}\left(1-\gamma_{(j)}^{(t) U}\right)^{w_{j}}}{\prod_{j=1}^{n}\left(1+(\xi-1) \gamma_{(j)}^{(t) U}\right)^{w_{j}}+(\xi-1) \prod_{j=1}^{n}\left(1-\gamma_{(j)}^{(t) U}\right)^{w_{j}}}\right]\right\},
\end{aligned}
$$

where $(j):\{1,2, \ldots, n\} \rightarrow\{1,2, \ldots, n\}$ is a permutation such that $\alpha_{(1)} \geq \alpha_{(2)} \geq \cdots \geq \alpha_{(n)}$, and $W=\left(w_{1}, w_{2}, \ldots, w_{n}\right)^{T}$ is the associated weight vector with $0 \leq w_{j} \leq 1, j=1,2, \ldots, n$ and $\sum_{j=1}^{n} w_{j}=1$.

Remark 18. If $\xi=1$, the I-IVHFHOWA operator is reduced to the following one:

$$
\begin{aligned}
& \operatorname{I-IVHFOWA}\left(\alpha_{1}, \alpha_{2}, \ldots, \alpha_{n}\right)=\bigcup_{t=1}^{l}\{[1 \\
& \left.\left.-\prod_{j=1}^{n}\left(1-\gamma_{(j)}^{(t) L}\right)^{w_{j}}, 1-\prod_{j=1}^{n}\left(1-\gamma_{(j)}^{(t) U}\right)^{w_{j}}\right]\right\},
\end{aligned}
$$

which is called the improved interval-valued hesitant fuzzy ordered weighted averaging (I-IVHFOWA) operator.

If $\xi=2$, the I-IVHFHOWA operator is reduced to the following one:

$$
\begin{aligned}
& \text { I-IVHFEOWA }\left(\alpha_{1}, \alpha_{2}, \ldots, \alpha_{n}\right) \\
& \quad=\bigcup_{t=1}^{l}\left\{\left[\frac{\prod_{j=1}^{n}\left(1+\gamma_{(j)}^{(t) L}\right)^{w_{j}}-\prod_{j=1}^{n}\left(1-\gamma_{(j)}^{(t) L}\right)^{w_{j}}}{\prod_{j=1}^{n}\left(1+\gamma_{(j)}^{(t) L}\right)^{w_{j}}+\prod_{j=1}^{n}\left(1-\gamma_{(j)}^{(t) L}\right)^{w_{j}}},\right.\right. \\
& \left.\left.\frac{\prod_{j=1}^{n}\left(1+\gamma_{(j)}^{(t) U}\right)^{w_{j}}-\prod_{j=1}^{n}\left(1-\gamma_{(j)}^{(t) U}\right)^{w_{j}}}{\prod_{j=1}^{n}\left(1+\gamma_{(j)}^{(t) U}\right)^{w_{j}}+\prod_{j=1}^{n}\left(1-\gamma_{(j)}^{(t) U}\right)^{w_{j}}}\right]\right\},
\end{aligned}
$$

which is called the improved interval-valued hesitant fuzzy Einstein ordered weighted averaging (I-IVHFEOWA) operator.

In the following, motivated by the geometric mean [45], we investigate the improved IVHFHOWG operator.

Definition 19. Let $\alpha_{j}=\left\{\left[\gamma_{j}^{L}, \gamma_{j}^{U}\right] \mid \gamma_{j} \in \alpha_{j}\right\}(j=1,2, \ldots, n)$ be a collection of IVHFEs; the improved IVHFHOWG (IIVHFHOWG) operator is a mapping I-IVHFHOWG $: H^{n} \rightarrow$ $H$, such that

$$
\begin{aligned}
& \operatorname{I-IVHFHOWG}\left(\alpha_{1}, \alpha_{2}, \ldots, \alpha_{n}\right)=\bigotimes_{j=1}^{n} \alpha_{(j)}^{w_{j}}=\bigcup_{t=1}^{l}\left\{\left[\frac{\xi \prod_{j=1}^{n}\left(\gamma_{(j)}^{(t) L}\right)^{w_{j}}}{\prod_{j=1}^{n}\left(1+(\xi-1)\left(1-\gamma_{(j)}^{(t) L}\right)\right)^{w_{j}}+(\xi-1) \prod_{j=1}^{n}\left(\gamma_{(j)}^{(t) L}\right)^{w_{j}}},\right.\right. \\
& \left.\left.\frac{\xi \prod_{j=1}^{n}\left(\gamma_{(j)}^{(t) U}\right)^{w_{j}}}{\prod_{j=1}^{n}\left(1+(\xi-1)\left(1-\gamma_{(j)}^{(t) U}\right)\right)^{w_{j}}+(\xi-1) \prod_{j=1}^{n}\left(\gamma_{(j)}^{(t) U}\right)^{w_{j}}}\right]\right\},
\end{aligned}
$$

where $(j):\{1,2, \ldots, n\} \rightarrow\{1,2, \ldots, n\}$ is a permutation such that $\alpha_{(1)} \geq \alpha_{(2)} \geq \cdots \geq \alpha_{(n)}$, and let $W=\left(w_{1}, w_{2}, \ldots, w_{n}\right)^{T}$ be the associated weight vector with $0 \leq w_{j} \leq 1, j=1,2, \ldots, n$ and $\sum_{j=1}^{n} w_{j}=1$.

Remark 20. If $\xi=1$, the I-IVHFHOWG operator is degenerated to the following:

$$
\begin{aligned}
& \operatorname{I-IVHFOWG}\left(\alpha_{1}, \alpha_{2}, \ldots, \alpha_{n}\right) \\
& \quad=\bigcup_{t=1}^{l}\left\{\left[\prod_{j=1}^{n}\left(\gamma_{(j)}^{(t) L}\right)^{w_{j}}, \prod_{j=1}^{n}\left(\gamma_{(j)}^{(t) U}\right)^{w_{j}}\right]\right\},
\end{aligned}
$$

which is called the improved interval-valued hesitant fuzzy ordered weighted geometric (I-IVHFOWG) operator.

If $\xi=2$, the I-IVHFHOWG operator is degenerated to the following operator:

$$
\begin{aligned}
& \text { I-IVHFEOWG }\left(\alpha_{1}, \alpha_{2}, \ldots, \alpha_{n}\right) \\
& \quad=\bigcup_{t=1}^{l}\left\{\left[\frac{2 \prod_{j=1}^{n}\left(\gamma_{(j)}^{(t) L}\right)^{w_{j}}}{\prod_{j=1}^{n}\left(2-\gamma_{(j)}^{(t) L}\right)^{w_{j}}+\prod_{j=1}^{n}\left(\gamma_{(j)}^{(t) L}\right)^{w_{j}}},\right.\right.
\end{aligned}
$$




$$
\left.\left.\frac{2 \prod_{j=1}^{n}\left(\gamma_{(j)}^{(t) U}\right)^{w_{j}}}{\prod_{j=1}^{n}\left(2-\gamma_{(j)}^{(t) U}\right)^{w_{j}}+\prod_{j=1}^{n}\left(\gamma_{(j)}^{(t) U}\right)^{w_{j}}}\right]\right\},
$$

which is called the improved interval-valued hesitant fuzzy Einstein ordered weighted geometric (I-IVHFEOWG) operator.

In what follows, we discuss some desirable properties of the I-IVHFHOWA operator and I-IVHFHOWG operator.
Theorem 21 (idempotency). Let $\alpha_{j}=\left\{\left[\gamma_{j}^{L}, \gamma_{j}^{U}\right] \mid \gamma_{j} \in \alpha_{j}\right\} \quad(j=$ $1,2, \ldots, n)$ be a collection of IVHFEs; if all $\alpha_{j}, j=1,2, \ldots, n$, are equal, that is, $\alpha_{j}=\alpha$ for all $j$, then

$$
\begin{aligned}
& \operatorname{I-IVHFHOWA}\left(\alpha_{1}, \alpha_{2}, \ldots, \alpha_{n}\right)=\alpha, \\
& \operatorname{I-IVHFHOWG}\left(\alpha_{1}, \alpha_{2}, \ldots, \alpha_{n}\right)=\alpha .
\end{aligned}
$$

Proof. Since $\alpha_{j}=\alpha$ for all $j$, that is, $\alpha_{1}=\alpha_{2}=\cdots=\alpha_{n}=\alpha=$ $\left\{\left[\gamma^{(1) L}, \gamma^{(1) U}\right],\left[\gamma^{(2) L}, \gamma^{(2) U}\right], \ldots,\left[\gamma^{(l) L}, \gamma^{(l) U}\right]\right\}$, then we have

$$
\begin{aligned}
& \operatorname{I-IVHFHOWA}\left(\alpha_{1}, \alpha_{2}, \ldots, \alpha_{n}\right)=\bigoplus_{j=1}^{n} w_{j} \alpha_{(j)}=\bigcup_{t=1}^{l}\left\{\left[\frac{\prod_{j=1}^{n}\left(1+(\xi-1) \gamma_{(j)}^{(t) L}\right)^{w_{j}}-\prod_{j=1}^{n}\left(1-\gamma_{(j)}^{(t) L}\right)^{w_{j}}}{\prod_{j=1}^{n}\left(1+(\xi-1) \gamma_{(j)}^{(t) L}\right)^{w_{j}}+(\xi-1) \prod_{j=1}^{n}\left(1-\gamma_{(j)}^{(t) L}\right)^{w_{j}}},\right.\right. \\
& \left.\left.\frac{\prod_{j=1}^{n}\left(1+(\xi-1) \gamma_{(j)}^{(t) U}\right)^{w_{j}}-\prod_{j=1}^{n}\left(1-\gamma_{(j)}^{(t) U}\right)^{w_{j}}}{\prod_{j=1}^{n}\left(1+(\xi-1) \gamma_{(j)}^{(t) U}\right)^{w_{j}}+(\xi-1) \prod_{j=1}^{n}\left(1-\gamma_{(j)}^{(t) U}\right)^{w_{j}}}\right]\right\} \\
& =\bigcup_{t=1}^{l}\left\{\left[\frac{\prod_{j=1}^{n}\left(1+(\xi-1) \gamma^{(t) L}\right)^{w_{j}}-\prod_{j=1}^{n}\left(1-\gamma^{(t) L}\right)^{w_{j}}}{\prod_{j=1}^{n}\left(1+(\xi-1) \gamma^{(t) L}\right)^{w_{j}}+(\xi-1) \prod_{j=1}^{n}\left(1-\gamma^{(t) L}\right)^{w_{j}}},\right.\right. \\
& \left.\left.\frac{\prod_{j=1}^{n}\left(1+(\xi-1) \gamma^{(t) U}\right)^{w_{j}}-\prod_{j=1}^{n}\left(1-\gamma^{(t) U}\right)^{w_{j}}}{\prod_{j=1}^{n}\left(1+(\xi-1) \gamma^{(t) U}\right)^{w_{j}}+(\xi-1) \prod_{j=1}^{n}\left(1-\gamma^{(t) U}\right)^{w_{j}}}\right]\right\} \\
& =\bigcup_{t=1}^{l}\left\{\left[\frac{\left(1+(\xi-1) \gamma^{(t) L}\right)^{\sum_{j=1}^{n} w_{j}}-\left(1-\gamma^{(t) L}\right)^{\sum_{j=1}^{n} w_{j}}}{\left(1+(\xi-1) \gamma^{(t) L}\right)^{\sum_{j=1}^{n} w_{j}}+(\xi-1) \cdot\left(1-\gamma^{(t) L}\right)^{\sum_{j=1}^{n} w_{j}}}, \frac{\left(1+(\xi-1) \gamma^{(t) U}\right)^{\sum_{j=1}^{n} w_{j}}-\left(1-\gamma^{(t) U}\right)^{\sum_{j=1}^{n} w_{j}}}{\left(1+(\xi-1) \gamma^{(t) U}\right)^{\sum_{j=1}^{n} w_{j}}+(\xi-1) \cdot\left(1-\gamma^{(t) U}\right)^{\sum_{j=1}^{n} w_{j}}}\right]\right\} \\
& =\bigcup_{t=1}^{l}\left\{\left[\frac{\left(1+(\xi-1) \gamma^{(t) L}\right)-\left(1-\gamma^{(t) L}\right)}{\left(1+(\xi-1) \gamma^{(t) L}\right)+(\xi-1) \cdot\left(1-\gamma^{(t) L}\right)}, \frac{\left(1+(\xi-1) \gamma^{(t) U}\right)-\left(1-\gamma^{(t) U}\right)}{\left(1+(\xi-1) \gamma^{(t) U}\right)+(\xi-1) \cdot\left(1-\gamma^{(t) U}\right)}\right]\right\}=\bigcup_{t=1}^{l}\left\{\left[\gamma^{(t) L}, \gamma^{(t) U}\right]\right\} \\
& =\left\{\left[\gamma^{(1) L}, \gamma^{(1) U}\right],\left[\gamma^{(2) L}, \gamma^{(2) U}\right], \ldots,\left[\gamma^{(l) L}, \gamma^{(l) U}\right]\right\}=\alpha .
\end{aligned}
$$

Similarly, we have I-IVHFHOWG $\left(\alpha_{1}, \alpha_{2}, \ldots, \alpha_{n}\right)=\alpha$, which completes the proof of Theorem 3.

It can be easily proved that the I-IVHFHOWA and IIVHFHOWG operators have the following property.

Theorem 22 (boundedness). Let $\alpha_{j}=\left\{\left[\gamma_{j}^{L}, \gamma_{j}^{U}\right]\right.$ । $\left.\gamma_{j} \in \alpha_{j}\right\}(j=1,2, \ldots, n)$ be a collection of IVHFEs, $\alpha^{L}=\bigcup_{t=1}^{l}\left\{\left[\min _{1 \leq j \leq n} \gamma_{j}^{(t) L}, \min _{1 \leq j \leq n} \gamma_{j}^{(t) U}\right]\right\}, \alpha^{U}=$ $\bigcup_{t=1}^{l}\left\{\left[\max _{1 \leq j \leq n} \gamma_{j}^{(t) L}, \max _{1 \leq j \leq n} \gamma_{j}^{(t) U}\right]\right\}$; then

$$
\begin{aligned}
& \alpha^{L} \leq I \text { IVHFHOWA }\left(\alpha_{1}, \alpha_{2}, \ldots, \alpha_{n}\right) \leq \alpha^{U}, \\
& \alpha^{L} \leq \operatorname{I-IVHFHOWG}\left(\alpha_{1}, \alpha_{2}, \ldots, \alpha_{n}\right) \leq \alpha^{U} .
\end{aligned}
$$

4.2. Relationship among the Improved Interval-Valued Hesitant Fuzzy Prioritized Aggregation Operators. In what follows, we analyze the relationship among the I-IVHFOWA operator, I-IVHFOWG operator, I-IVHFEOWA operator, and IIVHFEOWG operator.

In order to study the relationship among these intervalvalued hesitant fuzzy information aggregation operators, we first introduce the following lemmas.

Lemma 23 (see [45]). Let $x_{j}>0, w_{j}>0, j=1,2, \ldots, n$, and $\sum_{j=1}^{n} w_{j}=1$; then $\prod_{j=1}^{n} x_{j}^{w_{j}} \leq \sum_{j=1}^{n} w_{j} x_{j}$, with equality if and only if $x_{1}=x_{2}=\cdots=x_{n}$.

Lemma 24 (see [46]). Let $a_{j}, b_{j}, c_{j}, d_{j}(j=1,2, \ldots, n)$ be four collections of nonnegative numbers; if $a_{j}-b_{j}-c_{j}-d_{j}=0, j=$ $1,2, \ldots, n$, then

$$
\prod_{j=1}^{n} a_{j}-\prod_{j=1}^{n} b_{j}-\prod_{j=1}^{n} c_{j}-\prod_{j=1}^{n} d_{j} \geq 0 .
$$


Theorem 25. Let $\alpha_{j}=\left\{\left[\gamma_{j}^{L}, \gamma_{j}^{U}\right] \mid \gamma_{j} \in \alpha_{j}\right\}(j=1,2, \ldots, n)$ be a collection of IVHFEs and let $W=\left(w_{1}, w_{2}, \ldots, w_{n}\right)^{T}$ be the associated weight vector with $0 \leq w_{j} \leq 1, j=1,2, \ldots, n$, and $\sum_{j=1}^{n} w_{j}=1 ;$ then

$$
\begin{aligned}
& \operatorname{I-IVHFEOWA}\left(\alpha_{1}, \alpha_{2}, \ldots, \alpha_{n}\right) \\
& \quad \leq \operatorname{I-IVHFOWA}\left(\alpha_{1}, \alpha_{2}, \ldots, \alpha_{n}\right) .
\end{aligned}
$$

Proof. Since $\sum_{j=1}^{n} w_{j}=1$ and $\mathrm{w}_{j} \geq 0, j=1,2, \ldots, n$, and according to Lemma 23, for any $\gamma_{1} \in \alpha_{1}, \gamma_{2} \in \alpha_{2}, \ldots, \gamma_{n} \in \alpha_{n}$, we have

$$
\begin{aligned}
& \prod_{j=1}^{n}\left(1+\gamma_{(j)}^{(t) L}\right)^{w_{j}}+\prod_{j=1}^{n}\left(1-\gamma_{(j)}^{(t) L}\right)^{w_{j}} \\
& \quad \leq \sum_{j=1}^{n} w_{j}\left(1+\gamma_{(j)}^{(t) L}\right)+\sum_{j=1}^{n} w_{j}\left(1-\gamma_{(j)}^{(t) L}\right) \\
& \quad=\sum_{j=1}^{n} w_{j}\left(1+\gamma_{(j)}^{(t) L}+1-\gamma_{(j)}^{(t) L}\right)=2 .
\end{aligned}
$$

Then

$$
\begin{aligned}
& \frac{\prod_{j=1}^{n}\left(1+\gamma_{(j)}^{(t) L}\right)^{w_{j}}-\prod_{j=1}^{n}\left(1-\gamma_{(j)}^{(t) L}\right)^{w_{j}}}{\prod_{j=1}^{n}\left(1+\gamma_{(j)}^{(t) L}\right)^{w_{j}}+\prod_{j=1}^{n}\left(1-\gamma_{(j)}^{(t) L}\right)^{w_{j}}} \\
& \quad=1-\frac{2 \prod_{j=1}^{n}\left(1-\gamma_{(j)}^{(t) L}\right)^{w_{j}}}{\prod_{j=1}^{n}\left(1+\gamma_{(j)}^{(t) L}\right)^{w_{j}}+\prod_{j=1}^{n}\left(1-\gamma_{(j)}^{(t) L}\right)^{w_{j}}} \\
& \leq 1-\frac{2 \prod_{j=1}^{n}\left(1-\gamma_{(j)}^{(t) L}\right)^{w_{j}}}{2}=1-\prod_{j=1}^{n}\left(1-\gamma_{(j)}^{(t) L}\right)^{w_{j}} .
\end{aligned}
$$

Similarly, we also get that

$$
\begin{aligned}
& \frac{\prod_{j=1}^{n}\left(1+\gamma_{(j)}^{(t) U}\right)^{w_{j}}-\prod_{j=1}^{n}\left(1-\gamma_{(j)}^{(t) U}\right)^{w_{j}}}{\prod_{j=1}^{n}\left(1+\gamma_{(j)}^{(t) U}\right)^{w_{j}}+\prod_{j=1}^{n}\left(1-\gamma_{(j)}^{(t) U}\right)^{w_{j}}} \\
& \quad \leq 1-\prod_{j=1}^{n}\left(1-\gamma_{(j)}^{(t) U}\right)^{w_{j}} .
\end{aligned}
$$

Then according to Definitions 4 and 5, we know that the score function of I-IVHFEOWA $\left(\alpha_{1}, \alpha_{2}, \ldots, \alpha_{n}\right)$ is less than the score function of I-IVHFOWA $\left(\alpha_{1}, \alpha_{2}, \ldots, \alpha_{n}\right)$; that is,

$$
\begin{aligned}
& s\left(\operatorname{I} \text {-IVHFEOWA }\left(\alpha_{1}, \alpha_{2}, \ldots, \alpha_{n}\right)\right) \\
& \quad \leq s\left(\operatorname{I-IVHFOWA}\left(\alpha_{1}, \alpha_{2}, \ldots, \alpha_{n}\right)\right) .
\end{aligned}
$$

It follows that

$$
\begin{aligned}
& \operatorname{I-IVHFEOWA}\left(\alpha_{1}, \alpha_{2}, \ldots, \alpha_{n}\right) \\
& \quad \leq \operatorname{I-IVHFOWA}\left(\alpha_{1}, \alpha_{2}, \ldots, \alpha_{n}\right) .
\end{aligned}
$$

Theorem 26. Let $\alpha_{j}=\left\{\left[\gamma_{j}^{L}, \gamma_{j}^{U}\right] \mid \gamma_{j} \in \alpha_{j}\right\}(j=1,2, \ldots, n)$ be a collection of IVHFEs, let $W=\left(w_{1}, w_{2}, \ldots, w_{n}\right)^{T}$ be the associated weight vector with $0 \leq w_{j} \leq 1, j=1,2, \ldots, n$, and $\sum_{j=1}^{n} w_{j}=1 ;$ then

$$
\begin{aligned}
& I \text {-IVHFOWG }\left(\alpha_{1}, \alpha_{2}, \ldots, \alpha_{n}\right) \\
& \quad \leq \operatorname{I-IVHFEOWG}\left(\alpha_{1}, \alpha_{2}, \ldots, \alpha_{n}\right) .
\end{aligned}
$$

Proof. Since $\sum_{j=1}^{n} w_{j}=1$ and $w_{j} \geq 0, j=1,2, \ldots, n$, and according to Lemma 23, for any $\gamma_{1} \in \alpha_{1}, \gamma_{2} \in \alpha_{2}, \ldots, \gamma_{n} \in \alpha_{n}$, we have

$$
\begin{aligned}
& \prod_{j=1}^{n}\left(2-\gamma_{(j)}^{(t) L}\right)^{w_{j}}+\prod_{j=1}^{n}\left(\gamma_{(j)}^{(t) L}\right)^{w_{j}} \\
& \leq \sum_{j=1}^{n} w_{j}\left(2-\gamma_{(j)}^{(t) L}\right)+\sum_{j=1}^{n} w_{j} \gamma_{(j)}^{(t) L} \\
& \quad=\sum_{j=1}^{n} w_{j}\left(2-\gamma_{(j)}^{(t) L}+w_{j} \gamma_{(j)}^{(t) L}\right)=2 .
\end{aligned}
$$

Then

$$
\begin{gathered}
\frac{2 \prod_{j=1}^{n}\left(\gamma_{(j)}^{(t) L}\right)^{w_{j}}}{\prod_{j=1}^{n}\left(2-\gamma_{(j)}^{(t) L}\right)^{w_{j}}+\prod_{j=1}^{n}\left(\gamma_{(j)}^{(t) L}\right)^{w_{j}}} \\
\geq \frac{2 \prod_{j=1}^{n}\left(\gamma_{(j)}^{(t) L}\right)^{w_{j}}}{2}=\prod_{j=1}^{n}\left(\gamma_{(j)}^{(t) L}\right)^{w_{j}} .
\end{gathered}
$$

Similarly, we have

$$
\frac{2 \prod_{j=1}^{n}\left(\gamma_{(j)}^{(t) U}\right)^{w_{j}}}{\prod_{j=1}^{n}\left(2-\gamma_{(j)}^{(t) U}\right)^{w_{j}}+\prod_{j=1}^{n}\left(\gamma_{(j)}^{(t) U}\right)^{w_{j}}} \geq \prod_{j=1}^{n}\left(\gamma_{(j)}^{(t) U}\right)^{w_{j}} .
$$

According to Definitions 4 and 5, we obtain that the score function of I-IVHFOWG $\left(\alpha_{1}, \alpha_{2}, \ldots, \alpha_{n}\right)$ is less than the score function of I-IVHFEOWG $\left(\alpha_{1}, \alpha_{2}, \ldots, \alpha_{n}\right)$; that is,

$$
\begin{aligned}
& s\left(\operatorname{I}-\operatorname{IVHFOWG}\left(\alpha_{1}, \alpha_{2}, \ldots, \alpha_{n}\right)\right) \\
& \quad \leq s\left(\operatorname{I}-\operatorname{IVHFEOWG}\left(\alpha_{1}, \alpha_{2}, \ldots, \alpha_{n}\right)\right) .
\end{aligned}
$$

It follows that

$$
\begin{aligned}
& \text { I-IVHFOWG }\left(\alpha_{1}, \alpha_{2}, \ldots, \alpha_{n}\right) \\
& \quad \leq \operatorname{I-IVHFEOWG}\left(\alpha_{1}, \alpha_{2}, \ldots, \alpha_{n}\right) .
\end{aligned}
$$

Theorem 27. Let $\alpha_{j}=\left\{\left[\gamma_{j}^{L}, \gamma_{j}^{U}\right] \mid \gamma_{j} \in \alpha_{j}\right\}(j=1,2, \ldots, n)$ be a collection of IVHFEs and let $W=\left(w_{1}, w_{2}, \ldots, w_{n}\right)^{T}$ be the associated weight vector with $0 \leq w_{j} \leq 1, j=1,2, \ldots, n$, and $\sum_{j=1}^{n} w_{j}=1 ;$ then

$$
\begin{aligned}
& \operatorname{I-IVHFEOWA}\left(\alpha_{1}, \alpha_{2}, \ldots, \alpha_{n}\right) \\
& \quad \geq \operatorname{I-IVHFEOWG}\left(\alpha_{1}, \alpha_{2}, \ldots, \alpha_{n}\right) .
\end{aligned}
$$


Proof. Since $\sum_{j=1}^{n} w_{j}=1$ and $w_{j} \geq 0, j=1,2, \ldots, n$, and for any $\gamma_{1} \in \alpha_{1}, \gamma_{2} \in \alpha_{2}, \ldots, \gamma_{n} \in \alpha_{n}$, we have

$$
\begin{aligned}
& \frac{\prod_{j=1}^{n}\left(1+\gamma_{(j)}^{(t) L}\right)^{w_{j}}-\prod_{j=1}^{n}\left(1-\gamma_{(j)}^{(t) L}\right)^{w_{j}}}{\prod_{j=1}^{n}\left(1+\gamma_{(j)}^{(t) L}\right)^{w_{j}}+\prod_{j=1}^{n}\left(1-\gamma_{(j)}^{(t) L}\right)^{w_{j}}} \\
& -\frac{2 \prod_{j=1}^{n}\left(\gamma_{(j)}^{(t) L}\right)^{w_{j}}}{\prod_{j=1}^{n}\left(2-\gamma_{(j)}^{(t) L}\right)^{w_{j}}+\prod_{j=1}^{n}\left(\gamma_{(j)}^{(t) L}\right)^{w_{j}}} \\
& =\left(\prod_{j=1}^{n}\left(2+\gamma_{(j)}^{(t) L}-\left(\gamma_{(j)}^{(t) L}\right)^{2}\right)^{w_{j}}\right. \\
& -\prod_{j=1}^{n}\left(2-3 \gamma_{(j)}^{(t) L}+\left(\gamma_{(j)}^{(t) L}\right)^{2}\right)^{w_{j}} \\
& -\prod_{j=1}^{n}\left(\gamma_{(j)}^{(t) L}+\left(\gamma_{(j)}^{(t) L}\right)^{2}\right)^{w_{j}} \\
& \left.-3 \prod_{j=1}^{n}\left(\gamma_{(j)}^{(t) L}-\left(\gamma_{(j)}^{(t) L}\right)^{2}\right)^{w_{j}}\right) \times\left(\prod_{j=1}^{n}\left(1+\gamma_{(j)}^{(t) L}\right)^{w_{j}}\right. \\
& \left.+\prod_{j=1}^{n}\left(1-\gamma_{(j)}^{(t) L}\right)^{w_{j}}\right)^{-1} \times\left(\prod_{j=1}^{n}\left(2-\gamma_{(j)}^{(t) L}\right)^{w_{j}}\right. \\
& \left.+\prod_{j=1}^{n}\left(\gamma_{(j)}^{(t) L}\right)^{w_{j}}\right)^{-1}=\left(\prod_{j=1}^{n}\left(2+\gamma_{(j)}^{(t) L}-\left(\gamma_{(j)}^{(t) L}\right)^{2}\right)^{w_{j}}\right. \\
& -\prod_{j=1}^{n}\left(2-3 \gamma_{(j)}^{(t) L}+\left(\gamma_{(j)}^{(t) L}\right)^{2}\right)^{w_{j}} \\
& -\prod_{j=1}^{n}\left(\gamma_{(j)}^{(t) L}+\left(\gamma_{(j)}^{(t) L}\right)^{2}\right)^{w_{j}} \\
& \left.-\prod_{j=1}^{n}\left(3 \gamma_{(j)}^{(t) L}-3\left(\gamma_{(j)}^{(t) L}\right)^{2}\right)^{w_{j}}\right) \times\left(\prod_{j=1}^{n}\left(1+\gamma_{(j)}^{(t) L}\right)^{w_{j}}\right. \\
& \left.+\prod_{j=1}^{n}\left(1-\gamma_{(j)}^{(t) L}\right)^{w_{j}}\right)^{-1} \times\left(\prod_{j=1}^{n}\left(2-\gamma_{(j)}^{(t) L}\right)^{w_{j}}\right. \\
& \left.+\prod_{j=1}^{n}\left(\gamma_{(j)}^{(t) L}\right)^{w_{j}}\right)^{-1} \text {. }
\end{aligned}
$$

As $0 \leq \gamma_{j}^{(t) L} \leq \gamma_{j}^{(t) U} \leq 1, j=1,2, \ldots, n, t=1,2, \ldots, l$, then

$$
\begin{array}{r}
2+\gamma_{(j)}^{(t) L}-\left(\gamma_{(j)}^{(t) L}\right)^{2} \geq 0, \\
2-3 \gamma_{(j)}^{(t) L}+\left(\gamma_{(j)}^{(t) L}\right)^{2} \geq 0, \\
\gamma_{(j)}^{(t) L}+\left(\gamma_{(j)}^{(t) L}\right)^{2} \geq 0,
\end{array}
$$

$$
3 \gamma_{(j)}^{(t) L}-3\left(\gamma_{(j)}^{(t) L}\right)^{2} \geq 0
$$

$$
j=1,2, \ldots, n, t=1,2, \ldots, l .
$$

Note that

$$
\begin{gathered}
\left(2+\gamma_{(j)}^{(t) L}-\left(\gamma_{(j)}^{(t) L}\right)^{2}\right)-\left(2-3 \gamma_{(j)}^{(t) L}+\left(\gamma_{(j)}^{(t) L}\right)^{2}\right) \\
-\left(\gamma_{(j)}^{(t) L}+\left(\gamma_{(j)}^{(t) L}\right)^{2}\right)-\left(3 \gamma_{(j)}^{(t) L}-3\left(\gamma_{(j)}^{(t) L}\right)^{2}\right)=2 \\
+\gamma_{(j)}^{(t) L}-\left(\gamma_{(j)}^{(t) L}\right)^{2}-2+3 \gamma_{(j)}^{(t) L}-\left(\gamma_{(j)}^{(t) L}\right)^{2}-\gamma_{(j)}^{(t) L} \\
-\left(\gamma_{(j)}^{(t) L}\right)^{2}-3 \gamma_{(j)}^{(t) L}+3\left(\gamma_{(j)}^{(t) L}\right)^{2}=0, \\
j=1,2, \ldots, n, t=1,2, \ldots, l .
\end{gathered}
$$

Using Lemma 24 , for $t=1,2, \ldots, l$, we have

$$
\begin{aligned}
\prod_{j=1}^{n}( & \left.2+\gamma_{(j)}^{(t) L}-\left(\gamma_{(j)}^{(t) L}\right)^{2}\right)^{w_{j}} \\
& -\prod_{j=1}^{n}\left(2-3 \gamma_{(j)}^{(t) L}+\left(\gamma_{(j)}^{(t) L}\right)^{2}\right)^{w_{j}} \\
& -\prod_{j=1}^{n}\left(\gamma_{(j)}^{(t) L}+\left(\gamma_{(j)}^{(t) L}\right)^{2}\right)^{w_{j}} \\
& -\prod_{j=1}^{n}\left(3 \gamma_{(j)}^{(t) L}-3\left(\gamma_{(j)}^{(t) L}\right)^{2}\right)^{w_{j}} \geq 0 .
\end{aligned}
$$

It follows that

$$
\begin{aligned}
& \frac{\prod_{j=1}^{n}\left(1+\gamma_{(j)}^{(t) L}\right)^{w_{j}}-\prod_{j=1}^{n}\left(1-\gamma_{(j)}^{(t) L}\right)^{w_{j}}}{\prod_{j=1}^{n}\left(1+\gamma_{(j)}^{(t) L}\right)^{w_{j}}+\prod_{j=1}^{n}\left(1-\gamma_{(j)}^{(t) L}\right)^{w_{j}}} \\
& \quad-\frac{2 \prod_{j=1}^{n}\left(\gamma_{(j)}^{(t) L}\right)^{w_{j}}}{\prod_{j=1}^{n}\left(2-\gamma_{(j)}^{(t) L}\right)^{w_{j}}+\prod_{j=1}^{n}\left(\gamma_{(j)}^{(t) L}\right)^{w_{j}}} \geq 0 .
\end{aligned}
$$

Similarly, we have

$$
\begin{aligned}
& \frac{\prod_{j=1}^{n}\left(1+\gamma_{(j)}^{(t) U}\right)^{w_{j}}-\prod_{j=1}^{n}\left(1-\gamma_{(j)}^{(t) U}\right)^{w_{j}}}{\prod_{j=1}^{n}\left(1+\gamma_{(j)}^{(t) U}\right)^{w_{j}}+\prod_{j=1}^{n}\left(1-\gamma_{(j)}^{(t) U}\right)^{w_{j}}} \\
& \quad-\frac{2 \prod_{j=1}^{n}\left(\gamma_{(j)}^{(t) U}\right)^{w_{j}}}{\prod_{j=1}^{n}\left(2-\gamma_{(j)}^{(t) U}\right)^{w_{j}}+\prod_{j=1}^{n}\left(\gamma_{(j)}^{(t) U}\right)^{w_{j}}} \geq 0 .
\end{aligned}
$$

According to Definitions 4 and 5, the score function of I-IVHFEOWG $\left(\alpha_{1}, \alpha_{2}, \ldots, \alpha_{n}\right)$ is less than the score function of I-IVHFEOWA $\left(\alpha_{1}, \alpha_{2}, \ldots, \alpha_{n}\right)$; that is,

$$
\begin{aligned}
& s\left(\operatorname{I-IVHFEOWA}\left(\alpha_{1}, \alpha_{2}, \ldots, \alpha_{n}\right)\right) \\
& \quad \geq s\left(\operatorname{I-IVHFEOWG}\left(\alpha_{1}, \alpha_{2}, \ldots, \alpha_{n}\right)\right) .
\end{aligned}
$$


It follows that

$$
\begin{aligned}
& \operatorname{I-IVHFEOWA}\left(\alpha_{1}, \alpha_{2}, \ldots, \alpha_{n}\right) \\
& \quad \geq \operatorname{I-IVHFEOWG}\left(\alpha_{1}, \alpha_{2}, \ldots, \alpha_{n}\right) .
\end{aligned}
$$

According to Theorems 21-27, we have

Theorem 28. Let $\alpha_{j}=\left\{\left[\gamma_{j}^{L}, \gamma_{j}^{U}\right] \mid \gamma_{j} \in \alpha_{j}\right\}(j=1,2, \ldots, n)$ be a collection of IVHFEs and let $W=\left(w_{1}, w_{2}, \ldots, w_{n}\right)^{T}$ be the associated weight vector with $0 \leq w_{j} \leq 1, j=1,2, \ldots, n$, and $\sum_{j=1}^{n} w_{j}=1$; then

$$
\begin{aligned}
& \operatorname{I-IVHFOWG}\left(\alpha_{1}, \alpha_{2}, \ldots, \alpha_{n}\right) \\
& \leq I-I V H F E O W G\left(\alpha_{1}, \alpha_{2}, \ldots, \alpha_{n}\right) \\
& \leq I-\operatorname{IVHFEOWA}\left(\alpha_{1}, \alpha_{2}, \ldots, \alpha_{n}\right) \\
& \leq I-I V H F O W A\left(\alpha_{1}, \alpha_{2}, \ldots, \alpha_{n}\right) \text {. }
\end{aligned}
$$

\section{MAGDM Method under Interval-Valued Hesitant Fuzzy Environment}

Let $X=\left\{X_{1}, X_{2}, \ldots, X_{m}\right\}$ be a set of alternatives and let $C=\left\{C_{1}, C_{2}, \ldots, C_{n}\right\}$ be a collection of attributes. Suppose that the decision makers are required to provide not only the information that the alternative $X_{i}$ satisfies the attribute $C_{j}$ with anonymity, but also the information expressed by interval-valued values; then these values can be considered as an IVHFE $\alpha_{i j}$. When all the performances of the alternatives are provided, the interval-valued hesitant fuzzy decision matrix $D=\left(\alpha_{i j}\right)_{m \times n}$ can be constructed, where $\alpha_{i j}(i=$ $1,2, \ldots, m, j=1,2, \ldots, n)$ are in the form of IVHFEs. Assume that the weight vector of attribute is $W=\left(w_{1}, w_{2}, \ldots, w_{n}\right)^{\mathrm{T}}$, where $0 \leq w_{j} \leq 1, j=1,2, \ldots, n$, and $\sum_{j=1}^{n} w_{j}=1$.

5.1. The Approaches to Determine the Attribute Weights. In the following, we propose two approaches to determine the weight vector of attributes based on the continuous entropy.

Considering the entropy of the attribute $C_{j}$, the averaging entropy $E\left(C_{j}\right)$ of the attribute $C_{j}$ is given as

$$
E\left(C_{j}\right)=\frac{1}{m} \sum_{i=1}^{m} E\left(\alpha_{i j}\right)
$$

and each $E\left(\alpha_{i j}\right)$ can be calculated by (10).

According to the entropy theory, the entropy of an attribute is smaller across alternatives, which implies that it can provide decision makers with the effective information, and the attribute should be assigned a bigger weight.
In the process of decision making, sometimes, the information about attribute weights is completely unknown or incompletely known because of lack of knowledge or data, the influence of the decision environment, and the expert's limited expertise about the problem domain.

If the information about weight $w_{j}$ of the attribute $C_{j}, j=1,2, \ldots, n$ is completely unknown, we can establish the following equations for determining attribute weights:

$$
w_{j}=\frac{1-E\left(C_{j}\right)}{\sum_{j=1}^{n}\left(1-E\left(C_{j}\right)\right)}, \quad j=1,2, \ldots, n .
$$

If the information about weight $w_{j}$ of the attribute $C_{j}, j=$ $1,2, \ldots, n$ is incompletely known, in such a case, let $\Omega$ be the set of incomplete information about attribute weights; in order to get the optimal weight vector, the following model can be constructed:

$$
\begin{array}{ll}
(\mathrm{MOD}) \max & E_{W}=\sum_{j=1}^{n} w_{j}\left(1-E\left(C_{j}\right)\right) \\
\text { s.t. } & W \in \Omega, \\
& \sum_{j=1}^{n} w_{j}=1,
\end{array}
$$

5.2. The Procedure of Interval-Valued Hesitant Fuzzy $M A G D M$. From the above analysis, we investigate a MAGDM method under interval-valued hesitant fuzzy environment; the following steps are involved.

Step 1. If all the attributes $C_{j}, j=1,2, \ldots, n$ are of the benefit type, then the attribute values do not be normalized. Otherwise, we transform $D=\left(\alpha_{i j}\right)_{m \times n}$ into the normalized interval-valued hesitant fuzzy decision matrix $B=\left(\beta_{i j}\right)_{m \times n}$, where

$$
\begin{aligned}
& \beta_{i j}=\left\{\begin{array}{l}
\alpha_{i j}, \text { for benefit attribute } C_{j} \\
\alpha_{i j}^{c}, \text { for cost attribute } C_{j}, \\
\qquad i=1,2, \ldots, m, j=1,2, \ldots, n .
\end{array}\right.
\end{aligned}
$$

Step 2. Utilize (66) or model (67) to determine the weight vector of attribute: $W=\left(w_{1}, w_{2}, \ldots, w_{n}\right)^{T}$.

Step 3. Based on the interval-valued hesitant fuzzy decision matrix $B=\left(\beta_{i j}\right)_{m \times n}$, utilize the I-IVHFHOWA operator:

$$
\begin{aligned}
\beta_{i}= & \operatorname{I-IVHFHOWA}\left(\beta_{i 1}, \beta_{i 2}, \ldots, \beta_{i n}\right)=\bigoplus_{j=1}^{n} w_{j} \beta_{i(j)}=\bigcup_{t=1}^{l}\left\{\left[\frac{\prod_{j=1}^{n}\left(1+(\xi-1) \eta_{i(j)}^{(t) L}\right)^{w_{j}}-\prod_{j=1}^{n}\left(1-\eta_{i(j)}^{(t) L}\right)^{w_{j}}}{\prod_{j=1}^{n}\left(1+(\xi-1) \eta_{i(j)}^{(t) L}\right)^{w_{j}}-(\xi-1) \prod_{j=1}^{n}\left(1-\eta_{i(j)}^{(t) L}\right)^{w_{j}}},\right.\right. \\
& \left.\left.\frac{\prod_{j=1}^{n}\left(1+(\xi-1) \eta_{i(j)}^{(t) U}\right)^{w_{j}}-\prod_{j=1}^{n}\left(1-\eta_{i(j)}^{(t) U}\right)^{w_{j}}}{\prod_{j=1}^{n}\left(1+(\xi-1) \eta_{i(j)}^{(t) U}\right)^{w_{j}}-(\xi-1) \prod_{j=1}^{n}\left(1-\eta_{i(j)}^{(t) U}\right)^{w_{j}}}\right]\right\},
\end{aligned}
$$


TABLE 1: Interval-valued hesitant fuzzy decision matrix.

\begin{tabular}{cccccc}
\hline & $C_{1}$ & $C_{2}$ & $C_{3}$ & $C_{4}$ & $C_{5}$ \\
\hline$X_{1}$ & $\{[0.4,0.5],[0.3,0.4]\}$ & $\{[0.4,0.5],[0.2,0.3]\}$ & $\{[0.7,0.8],[0.5,0.6]\}$ & $\{[0.4,0.5],[0.4,0.4]\}$ & $\{[0.4,0.6],[0.4,0.5]\}$ \\
$X_{2}$ & $\{[0.5,0.6],[0.4,0.5]\}$ & $\{[0.5,0.7],[0.4,0.7]\}$ & $\{[0.6,0.8],[0.3,0.5]\}$ & $\{[0.8,0.9],[0.7,0.8]\}$ & $\{[0.5,0.5],[0.3,0.5]\}$ \\
$X_{3}$ & $\{[0.5,0.7],[0.3,0.4]\}$ & $\{[0.5,0.5],[0.3,0.4]\}$ & $\{[0.8,0.9],[0.7,0.8]\}$ & $\{[0.6,0.7],[0.5,0.7]\}$ & $\{[0.5,0.8],[0.5,0.7]\}$ \\
$X_{4}$ & $\{[0.5,0.8],[0.2,0.3]\}$ & $\{[0.4,0.7],[0.3,0.4]\}$ & $\{[0.6,0.8],[0.6,0.7]\}$ & $\{[0.5,0.6],[0.3,0.4]\}$ & $\{[0.5,0.6],[0.4,0.6]\}$ \\
\hline
\end{tabular}

or the I-IVHFHOWG operator:

$$
\begin{aligned}
& \beta_{i}=\operatorname{I-IVHFHOWG}\left(\beta_{i 1}, \beta_{i 2}, \ldots, \beta_{i n}\right)=\bigotimes_{j=1}^{n} \beta_{i(j)}^{w_{j}}=\bigcup_{t=1}^{l}\left\{\left[\frac{\xi \prod_{j=1}^{n}\left(\eta_{i(j)}^{(t) L}\right)^{w_{j}}}{\prod_{j=1}^{n}\left(1+(\xi-1)\left(1-\eta_{i(j)}^{(t) L}\right)\right)^{w_{j}}+(\xi-1) \prod_{j=1}^{n}\left(\eta_{i(j)}^{(t) L}\right)^{w_{j}}},\right.\right. \\
& \left.\left.\frac{\xi \prod_{j=1}^{n}\left(\eta_{i(j)}^{(t) U}\right)^{w_{j}}}{\prod_{j=1}^{n}\left(1+(\xi-1)\left(1-\eta_{i(j)}^{(t)}\right)\right)^{w_{j}}-(\xi-1) \prod_{j=1}^{n}\left(\eta_{i(j)}^{(t) U}\right)^{w_{j}}}\right]\right\},
\end{aligned}
$$

to aggregate the whole individual IVHFEs $\beta_{i j}(i=1,2$, $\ldots, m, j=1,2, \ldots, n)$ into the overall IVHFEs $\beta_{i}(i=$ $1,2, \ldots, m)$ of the alternatives $X_{i}$.

Step 4. Calculate the score functions $s\left(\beta_{i}\right)(i=1,2, \ldots, m)$ of the overall IVHFEs $\beta_{i}(i=1,2, \ldots, m)$ by Definition 5 .

Step 5. Rank the overall IVHFEs $\beta_{i}(i=1,2, \ldots, m)$ in accordance with Definition 5.

Step 6. Select the priority of the alternatives according to the ranking of $\beta_{i}(i=1,2, \ldots, m)$. Note that the bigger the $\beta_{i}$, the better the alternative $X_{i}$.

Step 7. End.

\section{Illustrative Example}

Emergency risk management (ERM) is a process which involves dealing with risks to the community arising from emergency events. Emergency management evaluation as one of the important parts of ERM aims at evaluating and improving social preparedness and organizational ability of an emergency operating center (EOC) in identifying, analyzing, and treating emergency risks to the community arising from emergency events [47, 48]. Fuzzy MAGDM method is widely used for emergency management evaluation, and there are some predetermined alternatives, associated with the attribute; based on these attributes, the evaluation result is to be made.

Suppose that there are four EOCs $X=\left\{X_{1}, X_{2}, X_{3}, X_{4}\right\}$ to be evaluated by evaluators, and the emergency management evaluation task has the following three features:

(1) There are five attributes to evaluate four EOCs, including $C_{1}$ : energy, $C_{2}$ : health and medical services,
$C_{3}$ : communication equipment, $C_{4}$ : emergency medical personnel, and $C_{5}$ : human resource.

(2) The attribute weight vector $W=\left(w_{1}, w_{2}, w_{3}, w_{4}, w_{5}\right)^{T}$ is completely unknown.

(3) The fuzzy decision matrix $D=\left(\alpha_{i j}\right)_{4 \times 5}$ is provided by evaluators, and the evaluated values are expressed by interval-valued hesitant fuzzy information, where $\alpha_{i j}(i=1,2,3,4, j=1,2, \ldots, 5)$ denotes the evaluated value of EOC $X_{i}$ under attribute $C_{j}$. The intervalvalued hesitant fuzzy decision matrix $D=\left(\alpha_{i j}\right)_{4 \times 5}$ is shown in Table 1.

The proposed MAGDM method is used to rank the EOCs and get the most desirable EOC. The following steps are involved.

Step 1. Considering that all the attributes $C_{j}(j=1,2, \ldots, 5)$ are the benefit attributes, then the attribute values are not normalized.

Step 2. Without loss of generality, take the BUM function $Q(y)=y$, and then $\lambda=0.5$ (medium value). For each attribute weight is completely unknown, we calculate the weight vector of attribute by the following equations:

$$
w_{j}=\frac{1-E\left(C_{j}\right)}{\sum_{j=1}^{5}\left(1-E\left(C_{j}\right)\right)}, \quad j=1,2, \ldots, 5 .
$$

By (10) and (65), we obtain the attribute weights as follows:

$$
\begin{aligned}
& w_{1}=0.0191, \\
& w_{2}=0.0424, \\
& w_{3}=0.1416, \\
& w_{4}=0.3774, \\
& w_{5}=0.4196 .
\end{aligned}
$$


Step 3. Let $\xi=1$ or 2 . Utilize the I-IVHFHOWA operator and the I-IVHFHOWG operator expressed by (69) and (70), respectively, to aggregate the all individual IVHFEs $\alpha_{i j}(j=$ $1,2, \ldots, 5)$ into the overall IVHFEs $\alpha_{i}(i=1,2,3,4)$ of the EOCs $X_{i}$, which are shown in Table 2 .

Step 4. Calculate the score functions $s\left(\alpha_{i}\right)(i=1,2,3,4)$ of the overall IVHFEs $\alpha_{i}(i=1,2,3,4)$ by Definition 5 , as shown in Table 3.

Step 5. Rank the overall IVHFEs $\alpha_{i}(i=1,2,3,4)$, which is shown in Table 4.

Step 6. Rank the EOCs.

According to the ordering of the overall IVHFEs $\alpha_{i}(i=$ $1,2,3,4)$, we can get the ranking of the EOCs, which are shown in Table 5. As can be seen, when we utilized the I-IVHFHOWA operator and the I-IVHFHOWG operator, respectively, the ranking of the EOCs is the same and the most desirable EOC is $X_{3}$.

Moreover, we can analyze how the different parameter value $\xi$ affects the rankings of the EOCs; in this case, we consider different values of $\xi$, which are provided by the decision makers. The results of collective overall preference values are shown in Figures 1-6. Figures 1 and 2 give the lower and upper limits of scores for EOCs, respectively, obtained by the I-IVHFHOWA operator, from which we can find that the lower and upper limits of scores for EOCs decrease as $\xi$ increases from 0 to 10 . If we use the I-IVHFHOWG operator instead of the I-IVHFHOWA operator to aggregate the attribute values, then the lower and upper limits of scores for EOCs can be found in Figures 3 and 4 . As the parameter value $\xi$ increases from 0 to 10 , we find that the lower and upper limits of scores for EOCs increase. Figure 5 illustrates the deviation values of lower limits of scores for EOCs obtained by I-IVHFHOWA operator and I-IVHFHOWG operator. Figure 6 illustrates the deviation values of upper limits of scores for EOCs obtained by I-IVHFHOWA operator and IIVHFHOWG operator. Figures 5 and 6 show that the scores for EOCs obtained by the I-IVHFHOWA operator are bigger than the ones obtained by the I-IVHFHOWG operator, and as the parameter value $\xi$ increases, we can find that the deviation decreases.

As can be seen, depending on the different parameter values $\xi$ used, the ranking of the EOCs does not change, and $Y_{5}$ is the best one.

In what follows, we apply the IVHFHOWG operator (6) proposed by Li and Peng [43] to deal with the aforementioned problem, and the ranking result and effectiveness will be compared with our proposed MAGDM approach. The following steps are involved.

Steps $1^{\prime}$ and $2^{\prime}$. See Steps 1 and 2.

Step $3^{\prime}$. Let $\xi=1$. By using the IVHFHOWG operator proposed by Li and Peng [43], we derive the overall IVHFEs $\alpha_{i}(i=1,2,3,4)$ of the EOCs $X_{i}$ as follows:

$$
\begin{aligned}
& \alpha_{1}=\{[0.6126,0.6790],[0.4204,0.6056] \text {, } \\
& \text { [0.4338, 0.5987], [0.4884, 0.6125], [0.6412, 0.7555], } \\
& \text { [0.6341, 0.7418], [0.4011, 0.5424], [0.5145, 0.6337], } \\
& \text { [0.4155, 0.6408], [0.3849, 0.5224], [0.4250, 0.5967], } \\
& \text { [0.6874, 0.7705], [0.6215, 0.7342], [0.5124, 0.6845], } \\
& \text { [0.5420, 0.6406], [0.4054, 0.6255], [0.6121, 0.6748], } \\
& \text { [0.5545, 0.5987], [0.4620, 0.5807], [0.5622, 0.6500], } \\
& \text { [0.5008, 0.6132] , [0.6414, 0.7120], [0.4111, 0.5865], } \\
& {[0.5215,0.6222],[0.4335,0.5619],[0.5656,0.6225] \text {, }} \\
& \text { [0.5525, 0.6254], [0.3994, 0.5488], [0.6215, 0.6782], } \\
& \text { [0.5303, 0.6245], [0.4664, 0.5440], } \\
& [0.5352,0.6357]\} \text {, } \\
& \alpha_{2}=\{[0.4329,0.5769],[0.3987,0.4595] \text {, } \\
& \text { [0.4227, 0.5454], [0.4007, 0.4668], [0.3654, 0.4333], } \\
& \text { [0.3848, 0.4411], [0.3999, 0.4765], [0.5145, 0.6337], } \\
& \text { [0.4155, 0.6408], [0.3849, 0.5224], [0.4250, 0.5967], } \\
& \text { [0.6874, 0.7705], [0.6215, 0.7342], [0.5124, 0.6845], } \\
& {[0.3615,0.4512],[0.3845,0.4778],[0.4213,0.5557] \text {, }} \\
& {[0.3390,0.4027],[0.3668,0.4750],[0.4343,0.5602] \text {, }} \\
& \text { [0.4517, 0.5710], [0.4225, 0.5419], [0.3688, 0.4627], } \\
& {[0.4115,0.5472],[0.3612,0.4470],[0.4519,0.5921] \text {, }} \\
& \text { [0.4697, 0.6002] , [0.4411, 0.5438], [0.4217, 0.5458], } \\
& \text { [0.3789, 0.4667], [0.4125, 0.5448], } \\
& [0.3860,0.4411]\} \text {, }
\end{aligned}
$$$$
\alpha_{3}=\{[0.5724,0.7563],[0.5081,0.6892] \text {, }
$$

[0.4343, 0.5663], [0.4333, 0.5779], [0.4003, 0.5217], $[0.3567,0.4764],[0.4102,0.4965],[0.5002,0.5844]$, $[0.4121,0.5117],[0.4090,0.5214],[0.4555,0.5658]$, [0.4452, 0.5543], [0.4300, 0.5617], [0.4221, 0.5388] , $[0.4076,0.4648],[0.4562,0.4948],[0.4150,0.5641]$, $[0.4127,0.5324],[0.4119,0.5600],[0.4553,0.5225]$, $[0.4411,0.5455],[0.6222,0.7017],[0.4518,0.5222]$, $[0.4333,0.5757],[0.4313,0.4924],[0.5124,0.6007]$, $[0.5399,0.6417],[0.5544,0.6488],[0.4335,0.5267]$, $[0.4149,0.5990],[0.5100,0.6254]$,

$[0.4322,0.5458]\}$, 


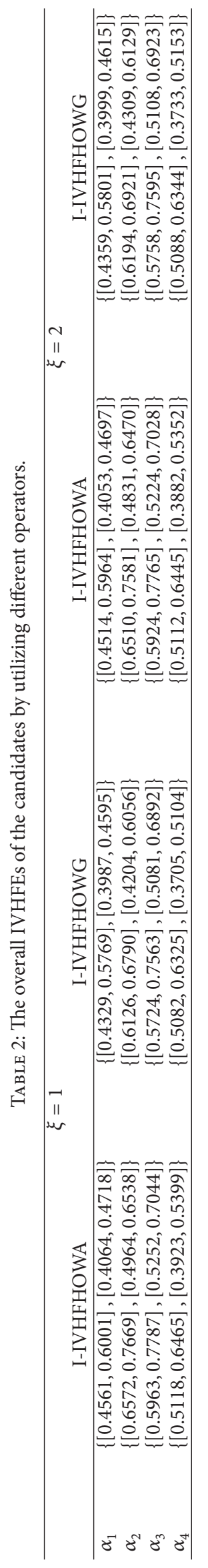


TABLE 3: The score functions obtained by the I-IVHFHOWA operator and I-IVHFHOWG operator.

\begin{tabular}{cccccc}
\hline & & $s\left(\alpha_{1}\right)$ & $s\left(\alpha_{2}\right)$ & $s\left(\alpha_{3}\right)$ & $s\left(\alpha_{4}\right)$ \\
\hline$\xi=1$ & I-IVHFHOWA & {$[0.4313,0.5760]$} & {$[0.5768,0.7103]$} & {$[0.5608,0.7416]$} & {$[0.4521,0.5932]$} \\
& I-IVHFHOWG & {$[0.4158,0.5182]$} & {$[0.5165,0.6423]$} & {$[0.5403,0.7227]$} & {$[0.4393,0.5715]$} \\
\multirow{2}{*}{$\xi=2$} & I-IVHFHOWA & {$[0.4284,0.5331]$} & {$[0.5671,0.7025]$} & {$[0.5574,0.7397]$} & {$[0.4497,0.5898]$} \\
& I-IVHFHOWG & {$[0.4179,0.5208]$} & {$[0.5252,0.6525]$} & {$[0.5433,0.7259]$} & {$[0.4411,0.5748]$} \\
\hline
\end{tabular}

TABLE 4: Ordering of the overall IVHFEs.

\begin{tabular}{lll}
\hline$\xi=1$ & & Ordering \\
\hline \multirow{3}{*}{$\xi=2$} & I-IVHFHOWA & $\alpha_{3}>\alpha_{2}>\alpha_{4}>\alpha_{1}$ \\
& I-IVHFHOWG & $\alpha_{3}>\alpha_{2}>\alpha_{4}>\alpha_{1}$ \\
& I-IVHFHOWA & $\alpha_{3}>\alpha_{2}>\alpha_{4}>\alpha_{1}$ \\
\hline
\end{tabular}

TABLE 5: Ordering of the EOCs.

\begin{tabular}{lll}
\hline & & \multicolumn{1}{c}{ Ordering } \\
\hline$\xi=1$ & I-IVHFHOWA & $X_{3}>X_{2}>X_{4}>X_{1}$ \\
& I-IVHFHOWA & $X_{3}>X_{2}>X_{4}>X_{1}$ \\
$\xi=2$ & I-IVHFHOWA & $X_{3}>X_{2}>X_{4}>X_{1}$ \\
& I-IVHFHOWA & $X_{3}>X_{2}>X_{4}>X_{1}$ \\
\hline
\end{tabular}

$$
\alpha_{4}=\{[0.5082,0.6325],[0.3705,0.5104] \text {, }
$$

[0.3661, 0.4949], [0.5121, 0.6242], [0.4313, 0.4966],

[0.3774, 0.4361] , [0.4216, 0.5100], [0.3942, 0.5233] ,

[0.3887, 0.4685] , [0.4175, 0.5120], [0.3770, 0.4500],

[0.3822, 0.5013] , [0.3923, 0.4578], [0.3666, 0.4557],

[0.3505, 0.4394], [0.3770, 0.4625], [0.4111, 0.5239],

[0.3405, 0.4299] , [0.3588, 0.4607], [0.3958, 0.5110],

[0.4332, 0.5102] [0.3925, 0.4698], [0.3668, 0.4445],

[0.3917, 0.5043] , [0.3778, 0.5520], [0.4219, 0.5476],

[0.4191, 0.6485], [0.5334, 0.6525], [0.3971, 0.5063],

[0.3862, 0.4557], [0.3993, 0.5128],

$[0.3430,0.4447]\}$.

Step $4^{\prime}$. Based on the above overall IVHFEs $\alpha_{i}(i=1,2,3,4)$ and Definition 5, we can calculate the score functions $s\left(\alpha_{i}\right)(i=1,2,3,4)$ as follows:

$$
\begin{aligned}
& s\left(\alpha_{1}\right)=[0.4227,0.5198], \\
& s\left(\alpha_{2}\right)=[0.5089,0.6780], \\
& s\left(\alpha_{3}\right)=[0.5641,0.6973], \\
& s\left(\alpha_{4}\right)=[0.4648,0.5466] .
\end{aligned}
$$

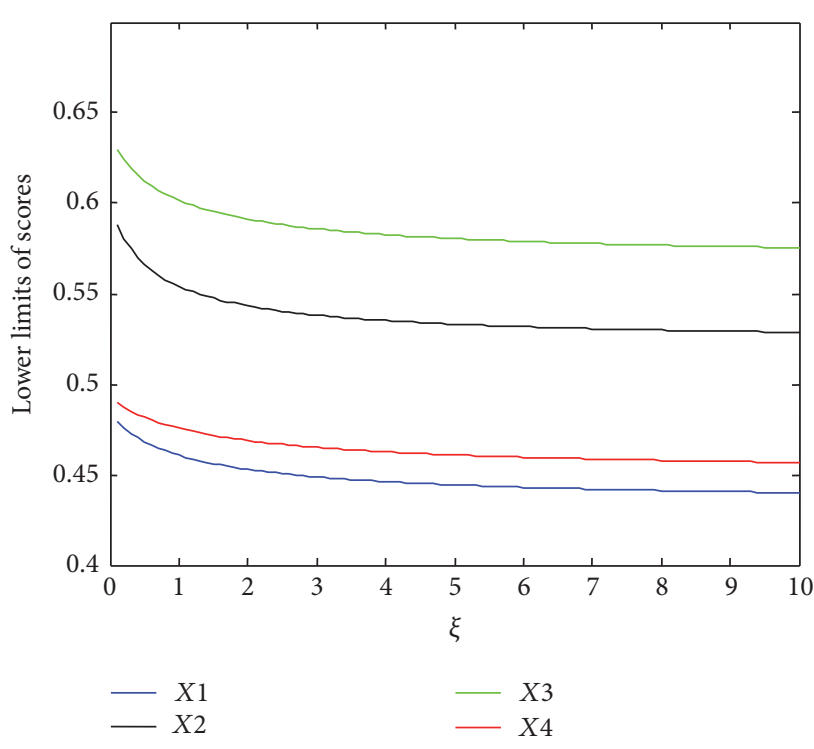

FIGURE 1: Lower limits of scores for EOCs obtained by IIVHFHOWA operator.

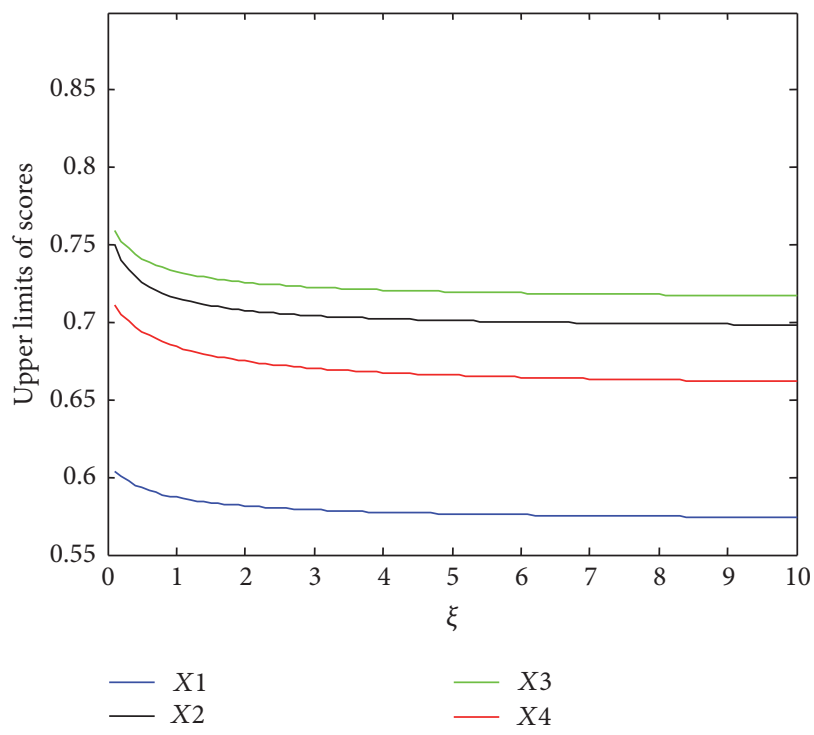

FIgURE 2: Upper limits of scores for EOCs obtained by IIVHFHOWA operator.

Step $5^{\prime}$. According to Definition 4 , we have $s\left(\alpha_{3}\right)>s\left(\alpha_{2}\right)>$ $s\left(\alpha_{4}\right)>s\left(\alpha_{1}\right)$; then the ranking order of the EOCs can be determined: that is, $\alpha_{3}>\alpha_{2}>\alpha_{4}>\alpha_{1}$, and the most desirable $\mathrm{EOC}$ is $X_{3}$. 


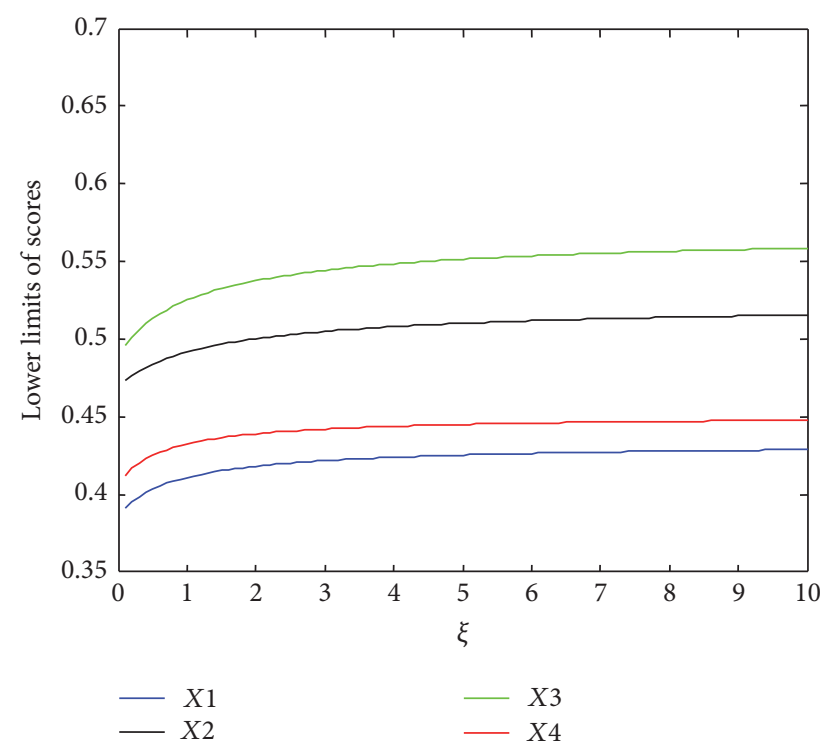

FIgURE 3: Lower limits of scores for EOCs obtained by IIVHFHOWG operator.

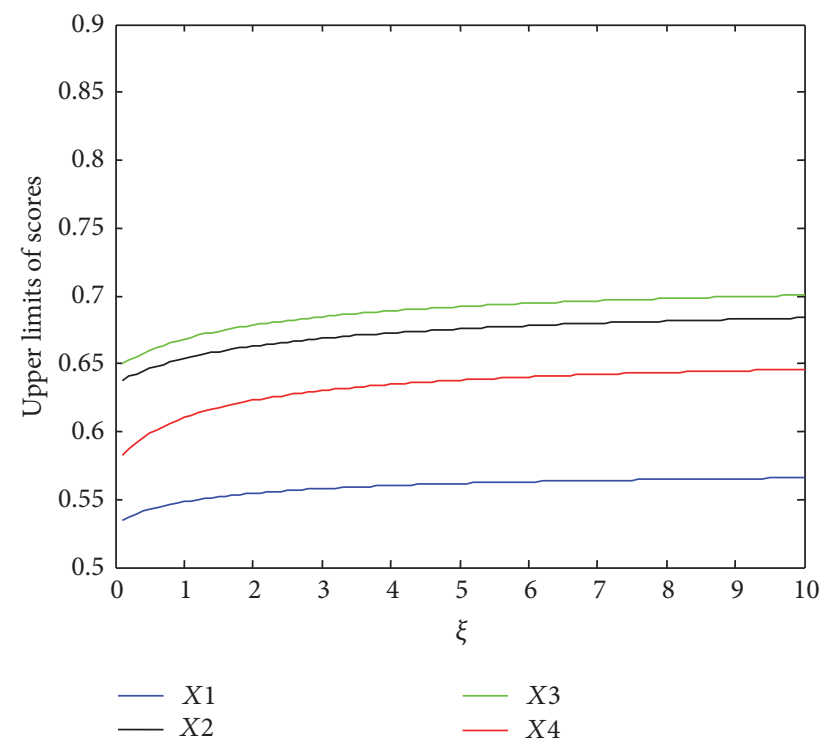

FIGURE 4: Upper limits of scores for EOCs obtained by IIVHFHOWG operator.

By using the proposed interval-valued hesitant fuzzy entropy formula, $\mathrm{Hu}$ and Zhou [49] developed a new approach for interval-valued hesitant fuzzy MAGDM problems. If we utilize the approach in [49] to cope with the aforementioned problem, then the main steps are follows.

Step $1^{\prime \prime}$. See Step 1.

Step $2^{\prime \prime}$. Because the attribute weight vector $W$ is completely unknown, by the equations

$$
w_{j}^{\prime}=\frac{1-E_{j}}{\sum_{j=1}^{5}\left(1-E_{j}\right)}, \quad j=1,2, \ldots, 5,
$$

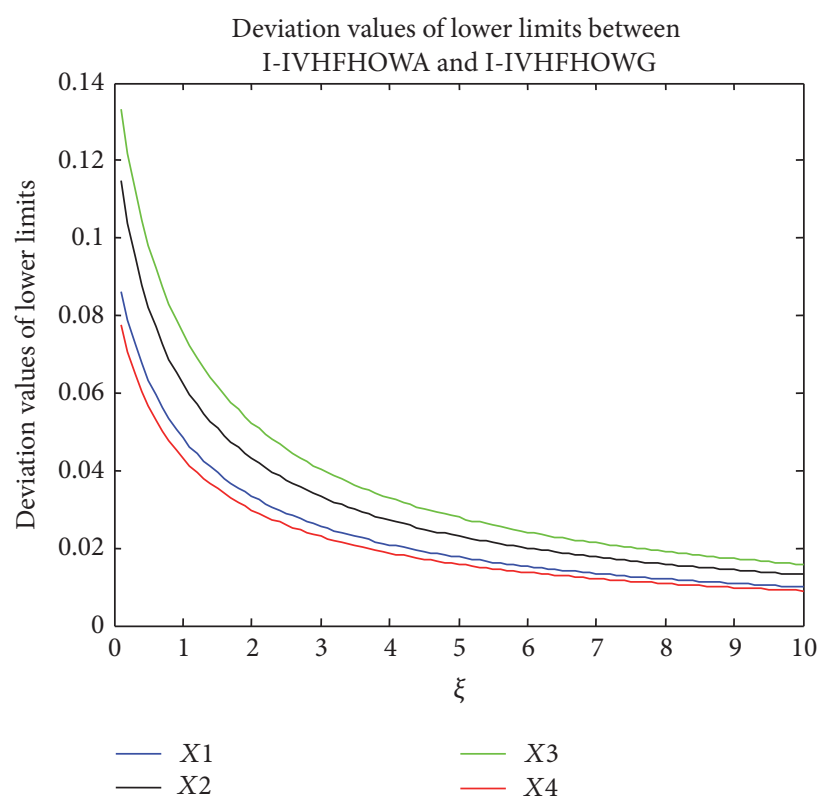

FIgURE 5: Deviation values of lower limits of scores for EOCs obtained by I-IVHFHOWA operator and I-IVHFHOWG operator.

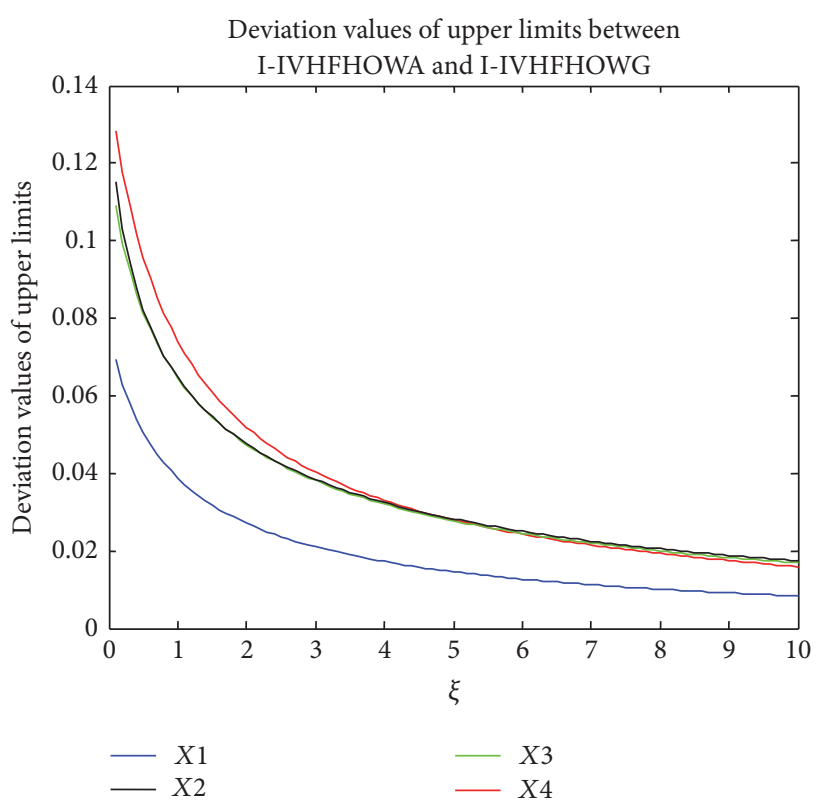

FIGURE 6: Deviation values of upper limits of scores for EOCs obtained by I-IVHFHOWA operator and I-IVHFHOWG operator.

where $E_{j}=(1 / m) \sum_{i=1}^{m} \widetilde{E}\left(\alpha_{i j}\right)$ and $\widetilde{E}\left(\alpha_{i j}\right)=(1 / l) \sum_{j=1}^{l}(1 /$ $\left.\left(2^{1-s}-1\right)\right)\left(\left(\left(\gamma^{(t) L}+\gamma^{(l-t+1) U}\right) / 2\right)^{s}+\left(1-\left(\gamma^{(t) L}+\gamma^{(l-t+1) U}\right) / 2\right)^{s}-1\right)$ (without loss of generality, let $s=2$ ), then we can calculate the attribute weights as follows:

$$
\begin{aligned}
& w_{1}^{\prime}=0.1147, \\
& w_{2}^{\prime}=0.1343, \\
& w_{3}^{\prime}=0.1806,
\end{aligned}
$$




$$
\begin{aligned}
& w_{4}^{\prime}=0.3449 \\
& w_{5}^{\prime}=0.2255
\end{aligned}
$$

Step $3^{\prime \prime}$. Utilizing the interval-valued hesitant fuzzy crossentropy equations (7) and (8) in [49], we obtain the positive cross-entropy $C_{i}^{+}(i=1,2,3,4)$ and negative cross-entropy $C_{i}^{-}(i=1,2,3,4)$ of each EOC: $C_{1}^{+}=0.5425, C_{1}^{-}=$ $0.5377, C_{2}^{+}=0.4201, C_{2}^{-}=0.4526, C_{3}^{+}=0.5119, C_{3}^{-}=$ $0.5833, C_{4}^{+}=0.6434, C_{24}^{-}=0.5770$.

Step $4^{\prime \prime}$. By (9) in [49], we determine the cross closeness degree $T_{i}(i=1,2,3,4)$ of the EOC $X_{i}$ :

$$
\begin{aligned}
& T_{1}=0.4978, \\
& T_{2}=0.5186, \\
& T_{3}=0.5326, \\
& T_{4}=0.4728 .
\end{aligned}
$$

Step $5^{\prime \prime}$. Since $T_{3}>T_{2}>T_{1}>T_{4}$, then the ranking of all the EOCs $X_{i}(i=1,2,3,4)$ is $X_{3}>X_{2}>X_{1}>X_{4}$, and the most desirable EOC is $X_{3}$.

Through the above example, we find that compared with the methods developed by Li and Peng [43] and Hu and Zhou [49] to rank the EOCs and determine the most desirable EOC, our proposed method has some advantages.

(1) Compared with the method developed by Li and Peng [43], although the developed method in this paper and that of Li and Peng [43] produce the same ranking of the four EOCs, that is, $X_{3}>X_{2}>X_{4}>X_{1}$, our proposed operators meet idempotency, and the decision making process of the information aggregation proposed by our method is more simplified than that of $\mathrm{Li}$ and Peng [43]. Therefore, the proposed MAGDM approach is both valid and practical to deal with group decision making problems.

(2) Comparing the four EOCs ranking derived by our approach with that of $\mathrm{Hu}$ and Zhou [49], we find that the method of $\mathrm{Hu}$ and Zhou [49] derives a little different ranking of these EOCs. However, from the original interval-valued hesitant fuzzy decision matrix $D=\left(\alpha_{i j}\right)_{4 \times 5}$, we find that most of the attribute values of EOC $X_{4}$ are greater than $X_{1}$, which means that $X_{4}$ is preferred to $X_{1}$. Therefore, our MAGDM approach is more rational than that of $\mathrm{Hu}$ and Zhou [49] in this case.

(3) In addition, we can consider a wide range of particular operators, including I-IVHFOWA operator, I-IVHFOWG operator, I-IVHFEOWA operator, and I-IVHFEOWG operator. The parameter $\xi$ can be viewed as the measure of the decision maker's attitudinal character; therefore, when people make a decision, they can choose the different parameter $\xi$ according to their own risk attitudes.

\section{Conclusions}

In FSs theory and modern decision making science in the uncertain environment, interval-valued hesitant fuzzy
MAGDM is an important research field. In this paper, we first presented the axiomatic definition of entropy for IVHFEs and constructed a continuous entropy formula for IVHFE to determine the entropy weights with respect to the decision matrix. Then, based on the Hamacher $t$-conorm and $t$-norm, we introduced some new adjusted operations on IVHFEs, and some improved interval-valued hesitant fuzzy Hamacher information aggregation operators have been investigated, such as I-IVHFHOWA operator and I-IVHFHOWG operator. Some of their desirable properties have been discussed. In addition, the relationship among the I-IVHFOWA operator, I-IVHFOWG operator, I-IVHFEOWA operator, and I-IVHFEOWG operator has also been analyzed. We also establish the programming model to determine optimal weight of attribute with the principle of minimum entropy. Finally, an approach to MAGDM has been developed, which is based on interval-valued hesitant fuzzy continuous entropy and the proposed operators. A numerical example for EOC selection is given to illustrate that the proposed aggregation methods provide a useful way to deal with MAGDM under interval-valued hesitant fuzzy environment.

However, there are still lots of work to be done in the future, including how to investigate sensitivity analysis and to give some practical applications of our method to the fields of decision making and supply chain management and medical diagnosis.

\section{Conflicts of Interest}

The authors declare that they have no conflicts of interest.

\section{Acknowledgments}

This work was supported by National Natural Science Foundation of China (no. 51204026) and Ministry of Public Security Key Laboratory of Building Fire Engineering Technology (no. KFKT2015ZD03).

\section{References}

[1] L. A. Zadeh, "Fuzzy sets," Information and Control, vol. 8, no. 3, pp. 338-353, 1965.

[2] K. T. Atanassov, "Intuitionistic fuzzy sets," Fuzzy Sets and Systems, vol. 20, no. 1, pp. 87-96, 1986.

[3] K. T. Atanassov, "More on intuitionistic fuzzy sets," Fuzzy Sets and Systems. An International Journal in Information Science and Engineering, vol. 33, no. 1, pp. 37-45, 1989.

[4] K. T. Atanassov, "Two theorems for intuitionistic fuzzy sets," Fuzzy Sets and Systems. An International Journal in Information Science and Engineering, vol. 110, no. 2, pp. 267-269, 2000.

[5] R. R. Yager, "Some aspects of intuitionistic fuzzy sets," Fuzzy Optimization and Decision Making. A Journal of Modeling and Computation Under Uncertainty, vol. 8, no. 1, pp. 67-90, 2009.

[6] P. Liu and F. Jin, "A multi-attribute group decision-making method based on weighted geometric aggregation operators of interval-valued trapezoidal fuzzy numbers," Applied Mathematical Modelling. Simulation and Computation for Engineering and Environmental Systems, vol. 36, no. 6, pp. 2498-2509, 2012.

[7] S. Abdullah, M. Aslam, and K. Hila, "Interval valued intuitionistic fuzzy sets in $\Gamma$-semihypergroups," International Journal of 
Machine Learning and Cybernetics, vol. 7, no. 2, pp. 217-228, 2016.

[8] M. Xia and Z. Xu, "Some new similarity measures for intuitionistic fuzzy values and their application in group decision making," Journal of Systems Science and Systems Engineering, vol. 19, no. 4, pp. 430-452, 2010.

[9] K. K. Myithili, R. Parvathi, and M. Akram, "Certain types of intuitionistic fuzzy directed hypergraphs," International Journal of Machine Learning and Cybernetics, vol. 7, no. 2, pp. 287-295, 2016.

[10] G. Wei, "Gray relational analysis method for intuitionistic fuzzy multiple attribute decision making," Expert Systems with Applications, vol. 38, no. 9, pp. 11671-11677, 2011.

[11] M. Xia and Z. S. Xu, "Entropy/cross entropy-based group decision making under intuitionistic fuzzy environment," Information Fusion, vol. 13, no. 1, pp. 31-47, 2012.

[12] B. Kang, Y. Hu, Y. Deng, and D. Zhou, "A new methodology of multicriteria decision-making in supplier selection based on $Z$-numbers," Mathematical Problems in Engineering, Article ID 8475987, Art. ID 8475987, 17 pages, 2016.

[13] K. Atanassov and G. Gargov, "Interval valued intuitionistic fuzzy sets," Fuzzy Sets and Systems, vol. 31, no. 3, pp. 343-349, 1989.

[14] V. Torra and Y. Narukawa, "On hesitant fuzzy sets and decision," in Proceedings of the IEEE International Conference on Fuzzy Systems, pp. 1378-1382, Jeju-do, Republic of Korea, August 2009.

[15] V. Torra, "Hesitant fuzzy sets," International Journal of Intelligent Systems, vol. 25, no. 6, pp. 529-539, 2010.

[16] I. B. Turksen, "Interval valued fuzzy sets based on normal forms," Fuzzy Sets and Systems, vol. 20, no. 2, pp. 191-210, 1986.

[17] N. Chen, Z. S. Xu, and M. M. Xia, "Interval-valued hesitant preference relations and their applications to group decision making," Knowledge-Based Systems, vol. 37, pp. 528-540, 2013.

[18] N. Chen, Z. Xu, and M. Xia, "Correlation coefficients of hesitant fuzzy sets and their applications to clustering analysis," Applied Mathematical Modelling. Simulation and Computation for Engineering and Environmental Systems, vol. 37, no. 4, pp. 2197-2211, 2013.

[19] M. Xia and Z. Xu, "Hesitant fuzzy information aggregation in decision making," International Journal of Approximate Reasoning, vol. 52, no. 3, pp. 395-407, 2011.

[20] R. R. Yager and D. P. Filev, "Induced ordered weighted averaging operators," IEEE Transactions on Systems, Man, and Cybernetics B: Cybernetics, vol. 29, no. 2, pp. 141-150, 1999.

[21] J. Y. Dong and S. P. Wan, "Arithmetic aggregation operators for interval-valued intuitionistic linguistic variables and application to multi-attribute group decision making," Iranian Journal of Fuzzy Systems, vol. 13, no. 1, pp. 1-23, 163, 2016.

[22] R. R. Yager, "On ordered weighted averaging aggregation operators in multicriteria decisionmaking," Institute of Electrical and Electronics Engineers. Transactions on Systems, Man, and Cybernetics, vol. 18, no. 1, pp. 183-190, 1988.

[23] E. K. Zavadskas, J. Antucheviciene, S. H. R. Hajiagha, and S. S. Hashemi, "The interval-valued intuitionistic fuzzy MULTIMOORA method for group decision making in engineering," Mathematical Problems in Engineering, vol. 2015, Article ID 560690, 13 pages, 2015.

[24] X. Liu and L. Chen, "On the properties of parametric geometric OWA operator," International Journal of Approximate Reasoning, vol. 35, no. 2, pp. 163-178, 2004.
[25] X. Liu, "On the properties of equidifferent OWA operator," International Journal of Approximate Reasoning, vol. 43, no. 1, pp. 90-107, 2006.

[26] P. D. Liu, L. He, and X. C. Yu, "Generalized hybrid aggregation operators based on the 2-dimension uncertain linguistic information for multiple attribute group decision making," Group Decision and Negotiation, vol. 25, no. 1, pp. 103-126, 2016.

[27] Y. Chu and P. Liu, "Some two-dimensional uncertain linguistic Heronian mean operators and their application in multipleattribute decision making," Neural Computing and Applications, vol. 26, no. 6, pp. 1461-1480, 2015.

[28] Z. Xu, "Intuitionistic fuzzy aggregation operators," IEEE Transactions on Fuzzy Systems, vol. 15, no. 6, pp. 1179-1187, 2007.

[29] Z. Xu and R. R. Yager, "Some geometric aggregation operators based on intuitionistic fuzzy sets," International Journal of General Systems, vol. 35, no. 4, pp. 417-433, 2006.

[30] G. Wei, X. Zhao, and R. Lin, "Some hesitant interval-valued fuzzy aggregation operators and their applications to multiple attribute decision making," Knowledge-Based Systems, vol. 46, pp. 43-53, 2013.

[31] J.-Q. Zhu, F. Fu, K.-X. Yin, J.-Q. Luo, and D. Wei, "Approaches to multiple attribute decision making with hesitant intervalvalued fuzzy information under correlative environment," Journal of Intelligent \& Fuzzy Systems, vol. 27, no. 2, pp. 1057-1065, 2014.

[32] H. H. Uber, "logische verknunpfungenn unssharfer Aussagen undderen Zugenhorige Bewertungsfunktione," in Progress in Cybernatics and Systems Research, Trappl, Klir, and Riccardi, Eds., pp. 276-288, Hemisphere, Washington, DC, 1978.

[33] C. Q. Tan, W. T. Yi, and X. H. Chen, "Hesitant fuzzy Hamacher aggregation operators for multicriteria decision making," Applied Soft Computing, vol. 26, pp. 325-349, 2015.

[34] Z. S. Xu and M. M. Xia, "Hesitant fuzzy entropy and crossentropy and their use in multiattribute decision-making," International Journal of Intelligent Systems, vol. 27, no. 9, pp. 799-822, 2012.

[35] L. A. Zadeh, "Probability measures of fuzzy events," Journal of Mathematical Analysis and Applications, vol. 23, pp. 421-427, 1968.

[36] A. De Luca and S. Termini, "A definition of a nonprobabilistic entropy in the setting of fuzzy sets theory," Information and Control, vol. 20, no. 4, pp. 301-312, 1972.

[37] E. Szmidt and J. Kacprzyk, "Entropy for intuitionistic fuzzy sets," Fuzzy Sets and Systems. An International Journal in Information Science and Engineering, vol. 118, no. 3, pp. 467-477, 2001.

[38] J. Ye, "Multicriteria fuzzy decision-making method using entropy weights-based correlation coefficients of intervalvalued intuitionistic fuzzy sets," Applied Mathematical Modelling. Simulation and Computation for Engineering and Environmental Systems, vol. 34, no. 12, pp. 3864-3870, 2010.

[39] C.-P. Wei, P. Wang, and Y.-Z. Zhang, "Entropy, similarity measure of interval-valued intuitionistic fuzzy sets and their applications," Information Sciences. An International Journal, vol. 181, no. 19, pp. 4273-4286, 2011.

[40] Z. S. Xu and Q. L. Da, “The uncertain OWA operator," International Journal of Intelligent Systems, vol. 17, no. 6, pp. 569-575, 2002.

[41] E. P. Klement and R. Mesiar, Logical, Algebraic, Analytic, and Probabilistic Aspects of Triangular Norms, Elsevier, New York, USA, 2005. 
[42] M. M. Xia, Z. S. Xu, and B. Zhu, "Some issues on intuitionistic fuzzy aggregation operators based on Archimedean t-conorm and t-norm," Knowledge-Based Systems, vol. 31, pp. 78-88, 2012.

[43] L.-G. Li and D.-H. Peng, "Interval-valued hesitant fuzzy Hamacher synergetic weighted aggregation operators and their application to shale gas areas selection," Mathematical Problems in Engineering, Article ID 181050, Art. ID 181050, 25 pages, 2014.

[44] R. R. Yager, "OWA aggregation over a continuous interval argument with applications to decision making," IEEE Transactions on Systems, Man, and Cybernetics B: Cybernetics, vol. 34, no. 5, pp. 1952-1963, 2004.

[45] Z. Xu, "On consistency of the weighted geometric mean complex judgement matrix in AHP," European Journal of Operational Research, vol. 126, no. 3, pp. 683-687, 2000.

[46] F. Jin, Z. Ni, and H. Chen, "Interval-valued hesitant fuzzy Einstein prioritized aggregation operators and their applications to multi-attribute group decision making," Soft Computing, vol. 20, no. 5, pp. 1863-1878, 2016.

[47] J. Dong, D. Y. Yang, and S. P. Wan, "Trapezoidal intuitionistic fuzzy prioritized aggregation operators and application to multi-attribute decision making," Iranian Journal of Fuzzy Systems, vol. 12, no. 4, pp. 1-32, 157, 2015.

[48] F. Jin, Z. Ni, H. Chen, and Y. Li, "Approaches to group decision making with intuitionistic fuzzy preference relations based on multiplicative consistency," Knowledge-Based Systems, vol. 97, pp. 48-59, 2016.

[49] G. Z. Hu and Z. G. Zhou, "Interval-valued hesitant fuzzy entropy and its application to local higher education development research," Computer Engineering and Applications, vol. 50, no. 23, pp. 26-30, 2014. 


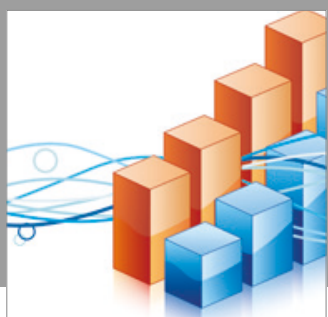

Advances in

Operations Research

vatersals

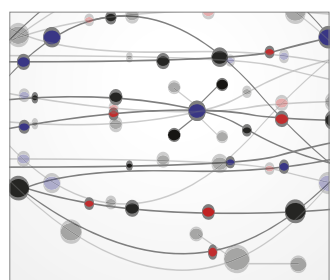

\section{The Scientific} World Journal
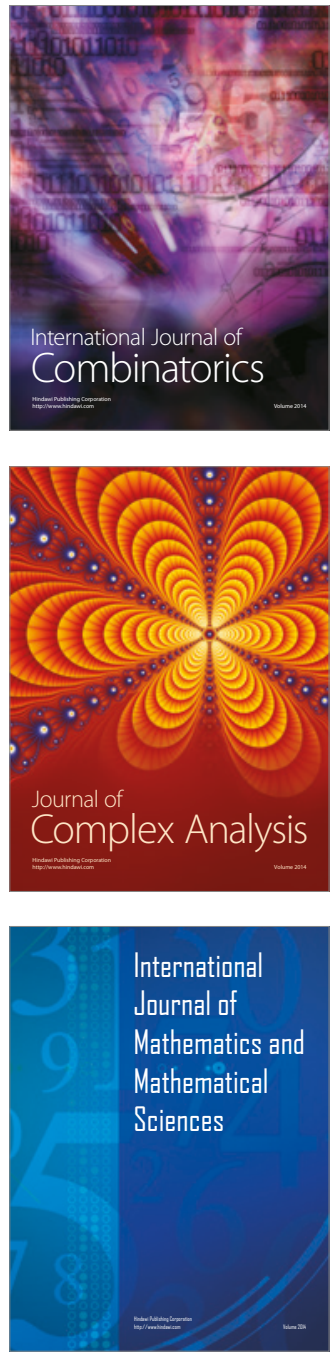
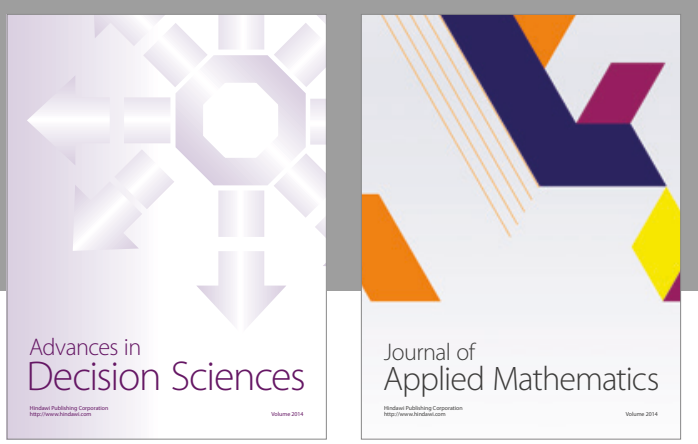

Algebra

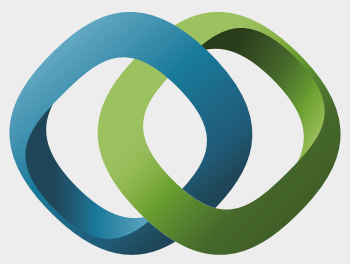

\section{Hindawi}

Submit your manuscripts at

https://www.hindawi.com
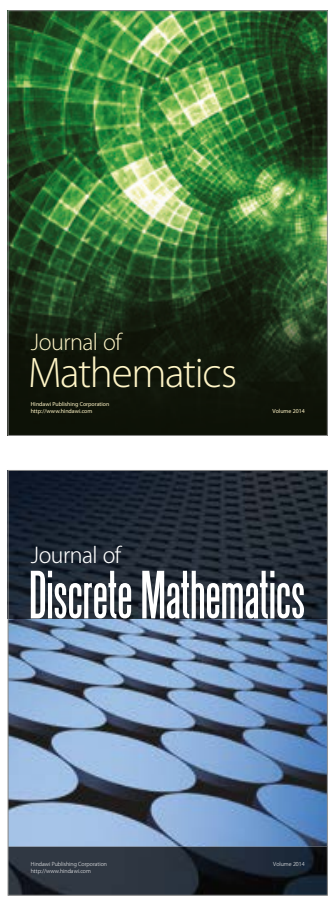

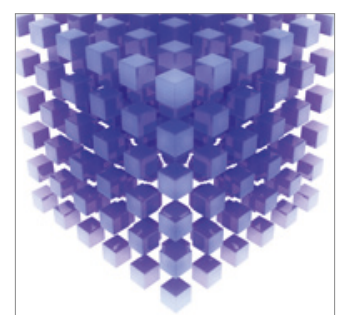

Mathematical Problems in Engineering
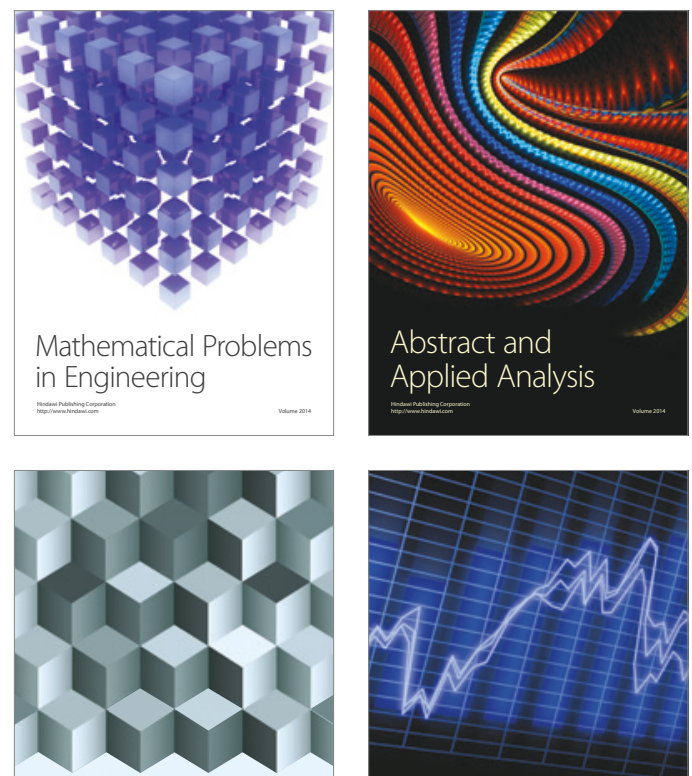

Journal of

Function Spaces

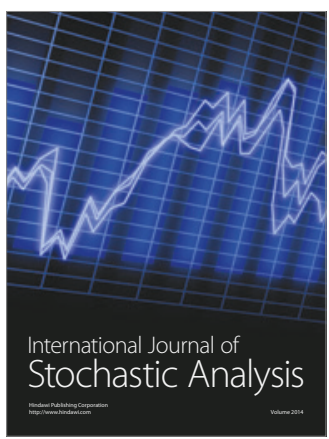

Probability and Statistics
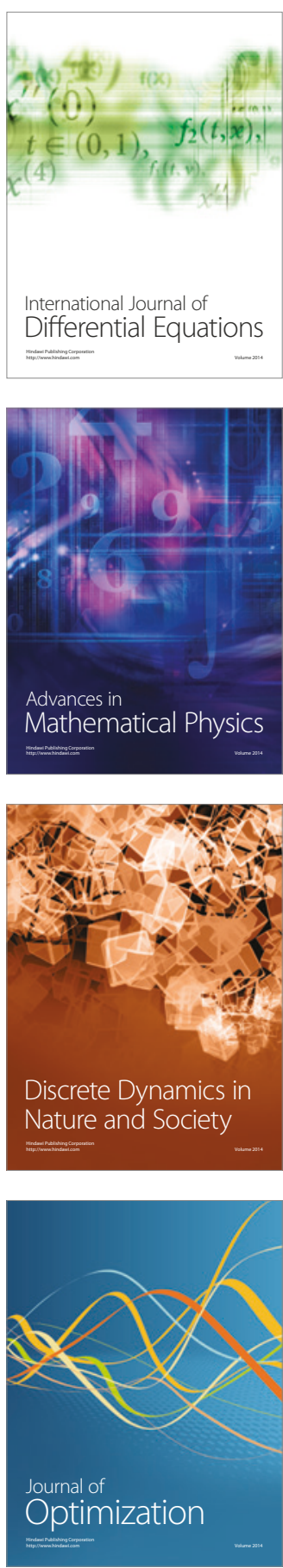